\title{
Abnormal sub-Riemannian geodesics: Morse index and rigidity
}

\author{
by \\ A. A. AGRACHEV $\left({ }^{1}\right)$ \\ Leading Research Associate, Steklov Mathematical Institute, \\ Russian Academy of Sciences, Vavilova ul. 42, 117966, Moscow, Russia; \\ E-mail: agrachev@de.mian.su. \\ and \\ A. V. SARYCHEV $\left({ }^{2}\right)$ \\ Invited Associate Professor, Departamento de Matemática, \\ Universidade de Aveiro, 3810, Aveiro, Portugal; E-mail: ansar@mat.ua.pt.
}

ABSTRACT. - Considering a smooth manifold $M$ provided with a subRiemannian structure, i.e. with Riemannian metric and nonintegrable vector distribution, we set a problem of finding for two given points $q^{0}, q^{1} \in M$ a length minimizer among Lipschitzian paths tangent to the vector distribution (admissible) and connecting these points. Extremals of this variational problem are called sub-Riemannian geodesics and we single out the abnormal sub-Riemannian geodesics, which correspond to the vanishing

( ${ }^{1}$ ) Partially supported by Russian Foundation for Fundamental Research; grant No. 93-011-1728.

$\left({ }^{2}\right)$ On leave from: Institute for Control Sciences, Russian Academy of Sciences, Moscow, Russia. The work was partially carried out when the author was visiting Twente University, Enschede, The Netherlands under financial support of Dutch Organization for Pure Research (NWO).

A.M.S. classifications: 58 A 30, 93 B 29.

Annales de l'Institut Henri Poincaré - Analyse non linéaire - 0294-1449

Vol. 13/96/06/\$ 7.00/

(C) 1996 L'Association Publications de l'Institut Henri Poincaré. Published by Elsevier B.V. All rights reserved 
Lagrange multiplier for the length functional. These abnormal geodesics are not related to the Riemannian structure but only to the vector distribution and, in fact, are singular points in the set of admissible paths connecting $q^{0}$ and $q^{1}$. Developing the Legendre-Jacobi-Morse-type theory of 2 nd variation for abnormal geodesics we investigate some of their specific properties such as weak minimality and rigidity-isolatedness in the space of admissible paths connecting the two given points.

Key words: Sub-Riemannan geometry, abnormal extremum.

RÉSUMÉ. - Soit $M$ une variété régulière avec une structure sousriemannienne (i.e. avec une métrique riemannienne et une distribution vectorielle non intégrable). Nous étudions l'existence d'un chemin de longueur minimale entre deux points $q^{0}$ et $q^{1}$ de $M$, parmi les chemins lipschitziens tangents à la distribution vectorielle (chemins admissibles). Des points stationnaires de ce problème variationnel sont appelés des géodésiques sous-riemanniennes et nous nous intéressons plus particulièrement aux géodésiques sous-riemanniennes « anormales ", correspondant à un multiplicateur de Lagrange nul. Les géodésiques anormales ne sont pas reliées à la structure riemannienne mais seulement à la distribution vectorielle et en fait sont des points irréguliers dans l'ensemble des chemins admissibles reliant $q^{0}$ à $q^{1}$. En développant une théorie de type Legendre-Jacobi-Morse de seconde variation pour les géodésiques anormales, nous étudions quelques-unes de leurs propriétés spécifiques comme la minimalité faible et la rigidité - le fait qu'elles sont isolées dans l'espace des chemins admissibles reliant les deux points.

\section{INTRODUCTION}

The paper deals with abnormal sub-Riemannian geodesics. Let us remind that a sub-Riemannian structure on a Riemannian manifold $M$ is given by a completely non-integrable (or completely non-holonomic, or possessing full Lie rank) vector distribution $\mathcal{D}$ on $M$. A locally Lipschitzian path $q(\tau) \in W_{\infty}^{1}[0, T]\left(W_{\infty}^{1}[0, T]\right.$ denotes the space of Lipschitzian paths $\tau \mapsto q(\tau)$ on $M)$ is called admissible if its tangents lie in $\mathcal{D}$ for almost all $\tau \in[0, T]$. Given two points $q^{0}$ and $q^{1}$ we set a problem of finding weakly (or equivalently $W_{\infty}^{1}$-locally) minimal admissible path connecting $q^{0}$ with $q^{1}$. 
The problem looks like direct generalization of the classical Riemannian case, but in fact there is an essential difference. Namely the space of all locally Lipschitzian paths, which connect $q^{0}$ and $q^{1}$, has natural structure of Banach manifold. Critical points of the length functional on this manifold are Riemannian geodesics and all paths of minimal length are among them. On the contrary the space of admissible paths, which connect $q^{0}$ and $q^{1}$, is not in general a manifold; it may have singularities. These singularities correspond to so called abnormal sub-Riemannian geodesics, which do not depend on Riemannian structure on $M$ and are completely determined by distribution $\mathcal{D}$.

The term "abnormal" comes from optimization theory, since the problem of finding minimal admissible path can be obviously reformulated as a Lagrange problem of Calculus of Variations. The extremals of the last problem are sub-Riemannian geodesics and, in particular, abnormal extremals, with vanishing Lagrange multiplier for the (length) functional, are abnormal sub-Riemannian geodesics.

There was a lot of activity tended to elimination of abnormal subRiemannian geodesics. Preprint [19] of R. Montgomery lists several (given by different authors) false proofs of the fact, that a minimal admissible path should correspond to some normal sub-Riemannian geodesic. The preprint contains also an important counterexample to this claim (see also [23]).

Main contribution of the paper is kind of Legendre-Jacobi-Morsetype theory of 2 nd variation for abnormal geodesics and derived from it necessary/sufficient conditions of weak minimality and rigidity, as well as existence of rigid paths of the distributions. Starting with the definition of 2 nd variation along an abnormal geodesic, we set 2 nd-order necessary/sufficient minimality conditions for abnormal geodesics. The conditions seem to be similar to the classic Legendre-Jacobi minimality conditions of Calculus of Variations, but not involving the length functional, they are appearances of different phenomenon, which means "degenerate" form of local minimality. Namely, the 2-nd order sufficient "minimality" condition imply rigidity of abnormal geodesic path, which is isolatedness up to reparametrization of this path in $W_{\infty}^{1}$-topology in the space of all admissible paths, which connect given end-points. Therefore the 2 nd-order necessary/sufficient minimality conditions are in fact necessary/sufficient rigidity conditions.

We go further and compute nullity and index of an abnormal geodesic, which are correspondingly dimension of the kernel and negative index of the 2 nd variation along the abnormal geodesic. This in particular enables us to verify the 2 nd-order rigidity conditions globally, on large time intervals. 
We use the index and nullity theorems to establish rigidity for several particular situations.

The paper is organized in following way. Section 2 contains preliminary material; of most importance for further presentation are some notations from chronological calculus and auxiliary results on symplectic geometry. In Section 3 we present Hamiltonian form of "geodesic equation" and introduce some invariants of geodesics. In Section 4 we introduce 1 st and $2 \mathrm{nd}$ variations along abnormal geodesics and define Morse index and nullity. Involving Goh and Generalized Legendre Condition along abnormal geodesics we derive (Theorem 4.4) a sufficient condition for smoothness of abnormal geodesic and announce (Theorems 4.1/4.8) necessary/sufficient conditions of rigidity. In Section 5 we introduce (Definition 5.1) Jacobi curve in Lagrangian Grassmanian for an abnormal geodesic and compute (Theorems 5.1 and 5.4) index and nullity of abnormal geodesics via symplectic invariants (Maslov-type indices) of the Jacobi curve. This enables us to establish (Theorem 5.5) local rigidity for abnormal geodesics meeting Goh and Strong Generalized Legendre Condition. In Section 6 we describe some class of distributions which do possess rigid abnormal geodesics (Theorem 6.1 and 6.2). In Section 7 we give more nice and simplified presentation of Legendre-Jacobi formalism for abnormal geodesics of 2-dimensional vector distributions. In Section 8 we investigate rigidity of trajectories for affine control systems (Theorems 8.5-8.9). In Appendix 1 (Section 9) we represent necessary/sufficient conditions (Theorems 9.1/9.5) for isolatedness of critical points of smooth mapping on critical level and use them to prove the necessary/sufficient conditions of rigidity for abnormal geodesics, which were established in the Section 4.

In Appendix 2 we prove the necessity of the (introduced in the Section 4) Goh and Generalized Legendre Condition for the finiteness of Morse index of abnormal geodesic (see the Proposition 4.3).

The presentation is self-contained, although we often refer to the papers [7], [8], which treats abnormal extrema for Lagrange problem of Calculus of Variations. One can find in that paper instructive analogies and details of some proofs.

In our work we were much inspired by a discussion on abnormal sub-Riemannian geodesics at the Conference "Geometric Methods in Nonlinear Optimal Control" (Sopron, Hungary, July 1991) and also by papers [11], [19] and discussions with M. Kawsky, R. Montgomery and H. J. Sussmann. The authors are grateful to H. J. Sussmann and the anonymous referee for valuable remarks regarding the manuscript, which we have taken into account when preparing the revised version. This paper 
was partially written when the second author was visiting the Faculty of Applied Mathematics at Twente University, Enschede, The Netherlands ; he is grateful to the faculty staff and especially to H. Nijmeijer and A. van der Schaft for hospitality.

\section{PRELIMINARIES}

In the paper we use notation and technical tools of chronological calculus developed by A. A. Agrachev and R. V. Gamkrelidze (see [5], [6]).

We will identify $C^{\infty}$ diffeomorphisms $P: M \longrightarrow M$ with automorphisms of the algebra $C^{\infty}(M)$ of smooth functions on $M$ : $\phi(\cdot) \mapsto P \phi=\phi(P(\cdot))$. The image of a point $q \in M$ under a diffeomorphism $P$ will be denoted by $q \circ P$.

Vector fields on $M$ are 1st-order differential operators on $M$ or arbitrary derivations of the algebra $C^{\infty}(M)$, i.e. R-linear mappings $X: C^{\infty}(M) \longrightarrow$ $C^{\infty}(M)$, satisfying the Leibnitz rule: $X(\alpha \beta)=(X \alpha) \beta+\alpha(X \beta)$. Value $X(q)$ of a vector field $X$ at a point $q \in M$ lies in the tangent space $\mathcal{T}_{q} M$ to the manifold $M$ at the point $q$. We denote by $\left[X^{1}, X^{2}\right]$ Lie bracket or commutator $X^{1} \circ X^{2}-X^{2} \circ X^{1}$ of vector fields $X^{1}, X^{2}$. It is again a 1st-order differential operator and in local coordinates on $M$ the Lie bracket can be presented as

$$
\begin{aligned}
{\left[X^{1}, X^{2}\right] } & =\left[\sum_{i=1}^{n} X_{i}^{1} \partial / \partial x_{i}, \sum_{i=1}^{n} X_{i}^{2} \partial / \partial x_{i}\right] \\
& =\sum_{i=1}^{n}\left(\partial X_{i}^{2} / \partial x X^{1}-\partial X_{i}^{1} / \partial x X^{2}\right) \partial / \partial x_{i}
\end{aligned}
$$

This operation introduces in the space of vector fields the structure of a Lie algebra denoted Vect $M$. For $X \in$ Vect $M$ we use the notation ad $X$ for the inner derivation of Vect $M:(\operatorname{ad} X) X^{\prime}=\left[X, X^{\prime}\right], \forall X^{\prime} \in$ Vect $M$.

For a diffeomorphism $P$ we use the notation $\operatorname{Ad} P$ for the following inner automorphism of the Lie algebra Vect $M: \operatorname{Ad} P X=P \circ X \circ P^{-1}=P_{*}^{-1} X$. The last notation stands for the result of translation of the vector field $X$ by the differential of the diffeomorphism $P^{-1}$.

To introduce topology in the space of vector fields and diffeomorphisms we start with a family of seminorms $\|\cdot\|_{s, K}$ in $C^{\infty}\left(R^{N}\right)$, where $s$ is a nonegative integer and $K \subset R^{N}$ is a compact. This family defines in $C^{\infty}\left(R^{N}\right)$ the topology of convergence of all derivatives on 
compacts. We call a family of functions $t \mapsto \varphi_{t},(t \in R)$ measurable if $\forall x \in R^{N} t \mapsto \varphi_{t}(x)$ is measurable. A measurable family is called locally integrable if $\forall s \geq 0, \forall K, \forall t_{1}, t_{2} \in R: \int_{t_{1}}^{t_{2}}\left\|\varphi_{t}\right\|_{s, K}<\infty$. A family $\omega_{t}$ is called absolutely continuous w.r.t. $t$ if $\omega_{t}=\omega_{t_{0}}+\int_{t_{0}}^{t} \varphi_{\tau} d \tau$ for some locally inegrable family $\varphi_{\tau}$. Since any manifold can be properly embedded into euclidean space of sufficiently large dimension $N$, one can introduce such a topology (independent on the embedding) in the space $C^{\infty}(M)$ of smooth functions on $M$.

As far as we treat the vector fields and the diffeomorphisms as operators on the $C^{\infty}(M)$ we may introduce the properties of local integrability or absolute continuity for parametrized by $t$ families of the operators in a weak sense (see [5] for details). Thus we call time-dependent vector field $t \mapsto X_{t}$ locally integrable if $t \mapsto X_{t} \varphi$ is locally integrable for any $\varphi \in C^{\infty}(M)$. From now on we assume all time-dependent vector fields to be locally integrable. A flow on $M$ is an absolutely continuous family $t \mapsto P_{t}$ of diffeomorphisms, satisfying the condition $P_{0}=I$ (where $I$ is the identity diffeomorphism). This means that $\forall \varphi \in C^{\infty}(M):\left(P_{t} \varphi\right)(q)=\varphi\left(P_{t}(q)\right)$ is absolutely continuous family of functions; $P_{0} \varphi=\varphi$.

A time-dependent vector field $X_{\tau}$ defines an ordinary differential equation $\dot{q}=X_{\tau}(q(\tau)), q(0)=q^{0}$ on the manifold $M$; if solutions of this differential equation exist for all $q^{0} \in M, \tau \in R$, then the vector field $X_{\tau}$ is called complete and defines a flow on $M$, being the unique solution of the (operator) differential equation:

$$
d P_{\tau} / d \tau=P_{\tau} \circ X_{\tau}, \quad P_{0}=I .
$$

This solution will be denoted by $P_{t}=\overrightarrow{\exp } \int_{0}^{t} X_{\tau} d \tau$, and is called (see [5], [6]) a right chronological exponential of $X_{\tau}$. If the vector field $X_{\tau} \equiv X$ is time-independent, then the corresponding flow is denoted by $P_{t}=e^{t X}$.

We introduce also Volterra expansion (or Volterra series) for the chronological exponential. It is (see [5], [6]):

$$
\overrightarrow{\exp } \int_{0}^{t} X_{\tau} d \tau \asymp I+\sum_{i=1}^{\infty} \int_{0}^{t} d \tau_{1} \int_{0}^{\tau_{1}} d \tau_{2} \ldots \int_{0}^{\tau_{i-1}} d \tau_{i}\left(X_{\tau_{i}} \circ \ldots \circ X_{\tau_{1}}\right) .
$$

We will need only the terms of zero-, first- and second-order in this expansion, which are

$$
\overrightarrow{\exp } \int_{0}^{t} X_{\tau} d \tau \asymp I+\int_{0}^{t} X_{\tau} d \tau+\int_{0}^{t} d \tau_{1} \int_{0}^{\tau_{1}} d \tau_{2}\left(X_{\tau_{2}} \circ X_{\tau_{1}}\right)+\cdots
$$


For time-independent $X$ one obtains

$$
e^{t X} \asymp I+t X+\left(t^{2} / 2\right) X \circ X+\cdots
$$

One more tool from chronological calculus is a "generalized variational formula" (see [5], [6] for its drawing):

$$
\overrightarrow{\exp } \int_{0}^{t}\left(\hat{X}_{\tau}+X_{\tau}\right) d \tau=\overrightarrow{\exp } \int_{0}^{t} \hat{X}_{\tau} d \tau \circ \overrightarrow{\exp } \int_{0}^{t} \operatorname{Ad}\left(\overrightarrow{\exp } \int_{t}^{\tau} \hat{X}_{\theta} d \theta\right) X_{\tau} d \tau
$$

Applying the operator $\operatorname{Ad}\left(\overrightarrow{\exp } \int_{0}^{\tau} \hat{X}_{\theta} d \theta\right)$ to a vector field $Y$ and differentiating

$\operatorname{Ad}\left(\overrightarrow{\exp } \int_{0}^{\tau} \hat{X}_{\theta} d \theta\right) Y=\left(\overrightarrow{\exp } \int_{0}^{\tau} \hat{X}_{\theta} d \theta\right) \circ Y \circ\left(\overrightarrow{\exp } \int_{0}^{\tau} \hat{X}_{\theta} d \theta\right)^{-1}$ w.r.t. $\tau$ one comes to the equality (see $[5,6])$ :

$$
\frac{d}{d \tau} \operatorname{Ad}\left(\overrightarrow{\exp } \int_{0}^{\tau} \hat{X}_{\theta} d \theta Y\right)=\operatorname{Ad}\left(\overrightarrow{\exp } \int_{0}^{\tau} \hat{X}_{\theta} d \theta\right) \operatorname{ad} \hat{X}_{\tau} Y
$$

which is of the same form as (2.1). Therefore $\operatorname{Ad}\left(\overrightarrow{\exp } \int_{0}^{\tau} \hat{X}_{\theta} d \theta\right)$ can be presented as an operator chronological exponential $\overrightarrow{\exp } \int_{0}^{t}$ ad $\hat{X}_{\theta} d \theta$ which for a time-independent vector field $\hat{X}_{\tau} \equiv \hat{X}$ is written as $e^{t \text { ad } \hat{X}}$. These exponentials also admit Volterra expansions:

$$
\begin{aligned}
& \underset{\exp }{\longrightarrow} \int_{0}^{t} \operatorname{ad} X_{\tau} d \tau \asymp I+\sum_{i=1}^{\infty} \int_{0}^{t} d \tau_{1} \int_{0}^{\tau_{1}} d \tau_{2} \ldots \\
& \times \int_{0}^{\tau_{i-1}} d \tau_{i}\left(\operatorname{ad} X_{\tau_{i}} \circ \cdots \operatorname{ad} X_{\tau_{1}} \asymp I+\int_{0}^{t} \operatorname{ad} X_{\tau} d \tau\right. \\
& \quad+\int_{0}^{t} d \tau_{1} \int_{0}^{\tau_{1}} d \tau_{2}\left(\operatorname{ad} X_{\tau_{2}} \circ \operatorname{ad} X_{\tau_{1}}\right)+\cdots
\end{aligned}
$$

and

$$
e^{t \operatorname{ad} X} \asymp I+t \operatorname{ad} X+\left(t^{2} / 2\right) \operatorname{ad} X \circ \text { ad } X+\cdots .
$$

In this new notation the generalized variational formula (2.4) can be rerepresented as:

$$
\begin{gathered}
\overrightarrow{\exp } \int_{0}^{t}\left(\hat{X}_{\tau}+X_{\tau}\right) d \tau=\overrightarrow{\exp } \int_{0}^{t} \hat{X}_{\tau} d \tau \circ \overrightarrow{\exp } \int_{0}^{t}\left(\overrightarrow{\exp } \int_{t}^{\tau} \operatorname{ad} \hat{X}_{\theta} d \theta\right) X_{\tau} d \tau \\
=\overrightarrow{\exp } \int_{0}^{t}\left(\overrightarrow{\exp } \int_{0}^{\tau} \operatorname{ad} \hat{X}_{\theta} d \theta\right) X_{\tau} d \tau \circ \overrightarrow{\exp } \int_{0}^{t} \hat{X}_{\tau} d \tau
\end{gathered}
$$


A vector distribution $\mathcal{D}$ on $M$ is a subbundle of tangent bundle $\mathcal{T} M$; a vector field $X$ is subjected to $\mathcal{D}$ if $X(q) \in \mathcal{D}_{q} \subset \mathcal{T}_{q} M$ for every $q \in M$. For a distribution $\operatorname{dim} \mathcal{D}_{q}$ does not change with $q \in M$.

Generalizations of vector distributions are differential systems or vector distributions with singularities $\left({ }^{3}\right)$ which are subbundles with nonconstant $\operatorname{dim} \mathcal{D}_{q}$. We call differential system any $C^{\infty}(M)$-submodule of Vect $M$; then vector distributions correspond to projective $C^{\infty}$-modules. Locally one may treat germ of vector distribution as free module.

If $\mathcal{D}$ is a differential system, then taking $C^{\infty}$-modules generated by Lie brackets of order $\leq k, k=1, \ldots$, of the vector fields subjected to $\mathcal{D}$ one obtains an expanding sequence of differential systems:

$$
\mathcal{D} \subseteq \mathcal{D}^{2}=[\mathcal{D}, \mathcal{D}] \cdots \subseteq \mathcal{D}^{k}=\left[\mathcal{D}, \mathcal{D}^{k-1}\right] \subseteq \cdots
$$

For any $q \in M$ the sequence of subspaces

$$
\mathcal{D}_{q} \subseteq \cdots \mathcal{D}_{q}^{k} \subseteq \mathcal{T}_{q} M
$$

is called flag of the differential system $\mathcal{D}$ at the point $q \in M$, while the sequence $n_{1}(q) \leq \cdots n_{k}(q) \leq \cdots$, where $n_{i}(q)=\operatorname{dim} \mathcal{D}_{q}^{i}$, is called growth vector of the differential system $\mathcal{D}$ at the point $q$. Differential system is called completely nonholonomic or having full Lie rank at a point $q \in M$ if $\mathcal{D}_{q}^{\bar{k}}=\mathcal{T}_{q} M$ for some $\bar{k}$. Differential system is called completely nonholonomic or having full Lie rank if for some $\bar{k} \mathcal{D}_{q}^{\bar{k}}=\mathcal{T}_{q} M$ for all $q \in M$.

If $\mathcal{D}$ is a distribution $\left(n_{1}(q) \equiv\right.$ const), then still $\mathcal{D}^{k}$ may lack to be distributions (may have singularities), since the growth vector of a distribution in general changes with $q$. Distribution is called regular if its growth vector is constant for all $q$.

We also have to introduce some notions of symplectic geometry (see [9], [14], [18] for more details). A symplectic structure in an even-dimensional linear space $\Sigma$ is defined by a nondegenerate bilinear skewsymmetric 2 -form $\sigma(\cdot, \cdot)$. Two vectors $\xi_{1}, \xi_{2} \in \Sigma$ are called skeworthogonal, written $\xi_{1} b \xi_{2}$, if $\sigma\left(\xi_{1}, \xi_{2}\right)=0$. If $N$ is a subspace of $\Sigma$, let us denote by $N^{b}$ its skeworthogonal complement: $N^{j}=\{\xi \in \Sigma \mid \sigma(\xi, \nu)=0, \forall \nu \in N\}$. Evidently $\operatorname{dim} N+\operatorname{dim} N^{b}=\operatorname{dim} \Sigma$. A subspace $\Gamma \subseteq \Sigma$ is called isotropic, when $\Gamma \subseteq \Gamma^{b}$, and coisotropic, when $\Gamma \supseteq \Gamma^{b}$. A subspace $\Lambda \subset \Sigma$ is called Lagrangian plane, when $\Lambda^{b}=\Lambda$. Such subspaces have dimension $\frac{1}{2} \operatorname{dim} \Sigma$.

$\left({ }^{3}\right)$ Not to be mixed with the differential systems determined by the differential forms; those have different kind of singularities. 
If $\Lambda$ is a Lagrangian plane and $\Gamma$ is isotropic, then it is easy to prove, that $\left(\Lambda \cap \Gamma^{b}\right)+\Gamma=(\Lambda+\Gamma) \cap \Gamma^{b}$ is a Lagrangian plane. We denote it by $\Lambda^{\Gamma}$.

The symplectic group $S p(\Sigma)$ is the group of those linear transformations of $\Sigma$, which preserve the symplectic form:

$$
S p(\Sigma)=S \in G L(\Sigma) \mid \sigma\left(S \xi_{1}, S \xi_{2}\right)=\sigma\left(\xi_{1}, \xi_{2}\right) \quad \forall \xi_{1}, \xi_{2} \in \Sigma .
$$

The elements of this group are called symplectic transformations of $\Sigma$. The Lie algebra of the symplectic group is:

$$
s p(\Sigma)=A \in g l(\Sigma) \mid \sigma\left(A \xi_{1}, \xi_{2}\right)=\sigma\left(A \xi_{2}, \xi_{1}\right) \quad \forall \xi_{1}, \xi_{2} \in \Sigma .
$$

Let $H$ be a real quadratic form on $\Sigma$ and $d_{\xi} H$ be the differential of $H$ at a point $\xi \in \Sigma$. Then $d_{\xi} H$ is a linear form on $\Sigma$ which depends linearly on $\xi$. For every $\xi \in \Sigma$ there exists a unique vector $\vec{H}(\xi) \in \Sigma$ which satisfies equality $\sigma(\vec{H}(\xi), \cdot)=d_{\xi} H$. It is easy to show that the linear operator $\vec{H}: \Sigma \rightarrow \Sigma$ belongs to $s p(\Sigma)$, and the mapping $H \mapsto \vec{H}$ is an isomorphism of the space of quadratic forms onto $s p(\Sigma)$. The differential equation $\dot{\xi}=\vec{H}(\xi)$ is called the linear Hamiltonian system corresponding to the quadratic Hamiltonian $H$.

Denote by $\mathcal{L}(\Sigma)$ the Grassmanian of Lagrangian subspaces of $\Sigma$. This is a smooth manifold of dimension $\frac{1}{8} \operatorname{dim} \Sigma(\operatorname{dim} \Sigma+2)$.

Certainly symplectic transformations transform Lagrangian planes into Lagrangian ones, hence the symplectic group acts on $\mathcal{L}(\Sigma)$. It is easy to show that it acts transitively.

Let us consider a tangent space $\mathcal{T}_{\Lambda} \mathcal{L}(\Sigma), \Lambda \in \mathcal{L}(\Sigma)$. Every quadratic form $h$ on $\Sigma$ corresponds to a linear Hamiltonian vector field $\vec{h}$ and a one-parameter subgroup $t \mapsto e^{t \vec{h}}$ in $S p(\Sigma)$. Let us consider the linear mapping

$$
h \mapsto d\left(e^{t \vec{h}} \Lambda\right) /\left.d t\right|_{t=0}
$$

of the space of quadratic forms to $\mathcal{T}_{\Lambda} \mathcal{L}(\Sigma)$. This mapping is surjective and its kernel consists of all quadratic forms which vanish on $\Lambda$. Thus two different quadratic forms correspond to the same vector from $\mathcal{T}_{\Lambda} \mathcal{L}(\Sigma)$ if and only if the restrictions of these forms on $\Lambda$ coincide. Hence we obtain a natural identification of the space $\mathcal{T}_{\Lambda} \mathcal{L}(\Sigma)$ with the space of quadratic forms on $\Lambda$.

A tangent vector $\eta \in \mathcal{T}_{\Lambda} \mathcal{L}(\Sigma)$ is called nonnegative if the corresponding quadratic form is nonnegative on $\Lambda$. An absolutely continuous curve $\Lambda_{\tau}(\tau \in[0, T])$ in $\mathcal{L}(\Sigma)$ is called nondecreasing if the velocities $\dot{\Lambda}_{\tau} \in \mathcal{T}_{\Lambda_{\tau}} \mathcal{L}(\Sigma)$ are nonnegative for almost all $\tau \in[0, T]$. 
Considering the action of symplectic group $S p(\Sigma)$ on $\mathcal{L}(\Sigma)$ one can easily verify, that pairs of Lagrangian planes $\left(\Lambda, \Lambda^{\prime}\right)$ have only one invariant w.r.t. this action: it is $\operatorname{dim}\left(\Lambda \cap \Lambda^{\prime}\right)$. For triples of Lagrangian planes, there are more invariants.

Let $\Lambda_{1}, \Lambda_{2}, \Lambda_{3}$ be Lagrangian planes. Let us present a vector $\lambda \epsilon$ $\left(\Lambda_{1}+\Lambda_{3}\right) \cap \Lambda_{2}$ as a sum $\lambda=\lambda_{1}+\lambda_{3}$ and consider on $\left(\Lambda_{1}+\Lambda_{3}\right) \cap \Lambda_{2}$ properly defined quadratic form $\beta(\lambda)=\sigma\left(\lambda_{1}, \lambda_{3}\right)$. Maslov index of the triple $\left(\Lambda_{1}, \Lambda_{2}, \Lambda_{3}\right)$ is signature of $\beta(\lambda)$. It is an invariant of the action of symplectic group.

In [1] a bit different invariant of a triple of Lagrangian planes $\left(\Lambda_{1}, \Lambda_{2}, \Lambda_{3}\right)$ was exploited for computation of Morse index for singular extremals.

DEFINITION 2.1. - Consider the quadratic form $\beta(\lambda)=\sigma\left(\lambda_{1}, \lambda_{3}\right)$ with the domain $\left(\left(\Lambda_{1}+\Lambda_{3}\right) \cap \Lambda_{2}\right) / \bigcap_{i=1}^{3} \Lambda_{i}$. A sum $\frac{1}{2} \operatorname{dim} \operatorname{ker} \beta+$ ind $^{-} \beta$, where ind $\beta$ is negative inertia index of $\beta$, is an invariant of the triple $\left(\Lambda_{1}, \Lambda_{2}, \Lambda_{3}\right)$ of Lagrangian planes. It is denoted by ind $\Lambda_{\Lambda_{2}}\left(\Lambda_{1}, \Lambda_{3}\right)$ and is called Maslov-type index.

Let us note, that $\operatorname{ker} \beta=\left(\left(\Lambda_{1} \cap \Lambda_{2}\right)+\left(\Lambda_{2} \cap \Lambda_{3}\right)\right) / \bigcap_{i=1}^{3} \Lambda_{i}$. We refer to [1] for a simple formula connecting this Maslov-type index with Maslov index of the triple and for the proof of the following "triangle inequality":

$$
\operatorname{ind}_{\Lambda_{0}}\left(\Lambda_{1}, \Lambda_{3}\right) \leq \operatorname{ind}_{\Lambda_{0}}\left(\Lambda_{1}, \Lambda_{2}\right)+\operatorname{ind}_{\Lambda_{0}}\left(\Lambda_{2}, \Lambda_{3}\right)
$$

It also follows directly from the definition, that

$$
\operatorname{ind}_{\Lambda_{1}}\left(\Lambda_{1}, \Lambda_{3}\right)=\frac{1}{2} \operatorname{dim} \operatorname{ker} \beta=\frac{1}{2}\left(\operatorname{dim} \Lambda_{1}-\operatorname{dim}\left(\Lambda_{1} \cap \Lambda_{3}\right)\right)
$$

A continuous curve $\Lambda(\tau) \in \mathcal{L}(\Sigma), 0 \leq \tau \leq 1$, is called simple if there exists $\Delta \in \mathcal{L}(\Sigma)$ such that $\Lambda(\tau) \cap \Delta=0 \forall \tau \in[0,1]$.

LEMma 2.1. - If $\Lambda(\tau) \in \mathcal{L}(\Sigma), 0 \leq \tau \leq 1$, is a simple nondecreasing curve in $\mathcal{L}(\Sigma)$, and $\Pi \in \mathcal{L}(\Sigma)$, then

$\operatorname{ind}_{\Pi}(\Lambda(0), \Lambda(1))=\operatorname{ind}_{\Pi}(\Lambda(0), \Lambda(\tau))+\operatorname{ind}_{\Pi}(\Lambda(\tau), \Lambda(1)), \forall \tau \in[0,1]$.

Lemma 2.2. - Let $\Lambda^{0}, \Lambda^{1} \in \mathcal{L}(\Sigma)$. There exist $\Delta \in \mathcal{L}(\Sigma)$ and neighborhoods $V^{0} \quad \ni \quad \Lambda^{0}, V^{1} \quad \ni \quad \Lambda^{1}$ in $\mathcal{L}(\Sigma)$ such that whenever $\Lambda \in V^{0}, \Lambda^{\prime} \in V^{1}$ and $\operatorname{dim}\left(\Lambda \cap \Lambda^{\prime}\right)=\operatorname{dim}\left(\Lambda^{0} \cap \Lambda^{1}\right)$ then there exists a simple nondecreasing curve $\Lambda(\tau), \tau \in[0,1]$ such that $\Lambda(0)=\Lambda, \Lambda(1)=$ $\Lambda^{\prime}, \Lambda(\tau) \cap \Delta=0 \forall \tau \in[0,1]$.

Both Lemmas are proved in [1]. 
DEFINITION 2.2. - Let $\Lambda(t), 0 \leq t \leq T$, be a nondecreasing curve in $\mathcal{L}(\Sigma)$ and $0=t_{0}<t_{1}<\cdots<t_{l}=T$ are such, that the curves $\left.\Lambda(\cdot)\right|_{\left[t_{i}, t_{i+1}\right]}, i=0, \ldots l-1$, are simple and $\Pi \in \mathcal{L}(\Sigma)$. The expression

$$
\operatorname{ind}_{\Pi} \Lambda(\cdot)=\sum_{i=0}^{l-1} \operatorname{ind}_{\Pi}\left(\Lambda\left(t_{i}\right), \Lambda\left(t_{i+1}\right)\right)
$$

is called Maslov index of the curve $\Lambda(t)$ with respect to $\Pi$.

It follows from the Lemma 2.1 that (2.10) does not depend on a choice of $t_{1}<\cdots<t_{l-1}$. If the curve $\Lambda(t)$ is closed $(\Lambda(0)=\Lambda(T))$, then $\operatorname{ind}_{\Pi} \Lambda(\cdot)$ does not depend also on the choice of $\Pi$ ( $c f .[1])$.

\section{NORMAL AND ABNORMAL GEODESICS. RIGIDITY}

The problem of finding minimal admissible path can be represented as following Lagrange problem of the Calculus of Variations with free final time:

$$
\begin{aligned}
\ell(T, u(\cdot)) & =\int_{0}^{T}\langle G(q(\tau)) u(\tau), G(q(\tau)) u(\tau)\rangle^{1 / 2} d \tau \longrightarrow \min \\
\dot{q} & =G(q) u, q(0)=q^{0}, u \in R^{r},\|u\|=1 \\
q(T) & =q^{1}
\end{aligned}
$$

Here $\langle\cdot, \cdot\rangle$ stays for the inner product in the tangent spaces $T_{q} M$; "control parameter" $u$ belongs to the $(r-1)$-dimensional unit sphere $S^{r-1}$; the controls $u(\tau)$ are measurable; $G(q)=\left(g^{1}(q), \ldots g^{r}(q)\right)$ is a $r$-tuple of smooth vector fields, which form a basis of the distribution $\mathcal{D}$. Since our consideration regards a small neighborhood of a nonselfintersecting path on $M$, then such basis can always be chosen.

We investigate problem of weak minimality, i.e. whether a given time $T$ and an admissible control $\hat{u}(\cdot)$ supply $\left(R \times L_{\infty}\right)$-local minimum for the problem (3.1)-(3.3).

Let us introduce classical 1st-order necessary condition of weak optimality for the Lagrange Problem of Calculus of Variations - Hamiltonian form of the Euler-Lagrange equation.

THEOREM 3.1. - If a pair $(T, \hat{u}(\cdot))$ is weak minimizer for the problem (3.1)-(3.3), i.e. corresponding trajectory $\hat{q}(\tau)(\tau \in[0, T])$ of (3.2) is 
$W_{\infty}^{1}$-locally minimal admissible path, then there exists a nonzero pair $\left(\hat{\psi}_{0}, \hat{\psi}(\cdot)\right)$, where $\hat{\psi}_{0}$ is a nonegative constant and $\hat{\psi}(\tau)$ is an absolutely continuous covector-function on $[0, T]$, such that $\hat{\psi}(\tau) \in \mathcal{T}_{\hat{q}(\tau)}^{*} M$ and the 5-tuple $\left(\hat{u}(\cdot), \hat{q}(\cdot), \hat{\psi}_{0}, \hat{\psi}(\cdot), T\right)$ :

1) satisfies in local coordinates on $M$ Hamiltonian system

$$
\begin{aligned}
\dot{q} & =\partial H / \partial \psi, \quad q(0)=q^{0}, \quad q(T)=q^{1} \\
\dot{\psi} & =-\partial H / \partial q,
\end{aligned}
$$

with a Hamiltonian

$$
H\left(u, q, \psi_{0}, \psi\right)=\hat{\psi}_{0}\langle G(q) u, G(q) u\rangle^{1 / 2}+\psi \cdot G(q) u
$$

2) meets stationarity condition

$$
\begin{gathered}
\left.\frac{\partial H}{\partial u}\right|_{\left(\hat{u}(\tau), \hat{q}(\tau), \hat{\psi}_{0}, \hat{\psi}(\tau)\right)} v=0, \quad \forall v \in T_{\hat{u}(\tau)} S^{r-1}, \\
\text { for almost all } \tau \in[0, T]
\end{gathered}
$$

and "transversality condition"

$$
H\left(\hat{u}(\tau), \hat{q}(\tau), \hat{\psi}_{0}, \hat{\psi}(\tau)\right)=0, \text { for almost all } \tau \in[0, T]
$$

DEFINITION 3.1. - Sub-Riemannian geodesic is an extremal of the Lagrange problem (3.1)-(3.3), i.e. a 5-tuple $\left(\hat{u}(\cdot), \hat{q}(\cdot), \hat{\psi}_{0}, \hat{\psi}(\cdot), T\right)$ meeting the conditions of the Theorem 3.1. Sub-Riemannian geodesic is called normal, if $\hat{\psi}_{0} \neq 0$, and abnormal, if $\hat{\psi}_{0}=0$. The corresponding triple $(\hat{u}(\cdot), \hat{q}(\cdot), T)$ is called sub-Riemannian geodesic path.

Remark. - Obviously any restriction $\left(\left.\hat{u}(\cdot)\right|_{[0, t]},\left.\hat{q}(\cdot)\right|_{[0, t]}, \hat{\psi}_{0},\left.\hat{\psi}(\cdot)\right|_{[0, t]}, t\right)$ of normal or abnormal sub-Riemannian geodesic $\left(\hat{u}(\cdot), \hat{q}(\cdot), \hat{\psi}_{0}, \hat{\psi}(\cdot), T\right)$ its restriction $\left(\left.\hat{u}(\cdot)\right|_{[0, t]},\left.\hat{q}(\cdot)\right|_{[0, t]}, \hat{\psi}_{0},\left.\hat{\psi}(\cdot)\right|_{[0, t]}, t\right)$ to a subinterval $[0, t] \subset[0, T]$ is also normal or abnormal sub-Riemannian geodesic correspondingly.

Remark. - A geodesic path $(\hat{u}(\cdot), \hat{q}(\cdot), T)$ may correspond to different geodesics with different $\hat{\psi}_{0}, \hat{\psi}(\cdot)$.

DEFINITION 3.2. - A corank of a geodesic path $(\hat{u}(\cdot), \hat{q}(\cdot), T)$ is dimension of the space of pairs $\left(\hat{\psi}_{0}, \hat{\psi}(\cdot)\right)$, which together with $(\hat{u}(\cdot), \hat{q}(\cdot), T)$ satisfy Theorem 3.1.

Definition 3.3. - A geodesic path $(\hat{u}(\cdot), \hat{q}(\cdot), T)$ is called corank $k$ abnormal geodesic path if the space of pairs $(0, \hat{\psi}(\cdot))$, which together with $(\hat{u}(\cdot), \hat{q}(\cdot), T)$ satisfy the Theorem 3.1 , is k-dimensional. 
Remark. - One should take precautions, when determining corank of abnormal geodesic path, since in a $k$-dimensional linear space of pairs $\left(\hat{\psi}_{0}, \hat{\psi}(\cdot)\right)$ there is $k$ - or $(k-1)$-dimensional subspace of pairs with vanishing $\psi_{0}$. Therefore it may happen, that corank $k$ geodesic path is corank $(k-1)$ abnormal geodesic path.

Whenever geodesic is abnormal, then the length functional $\ell$ does not enter the minimality conditions, given by the Theorem 3.1. No surprise that corresponding geodesic paths have not too much to do with the subRiemannian metric and minimality of length. It turns out that they often exhibit a phenomenon called in [25] rigidity.

Definition 3.4. - An admissible path $q(\cdot)$ of the vector distribution $\mathcal{D}$ with end-points $q^{0}$ and $q^{1}$ is called rigid if it is isolated up to a reparametrization in the metric of $W_{\infty}^{1}$ in the set $\mathcal{P}_{q^{0}}^{q^{1}}$ of all admissible paths, which connect $q^{0}$ and $q^{1}$.

Rigid admissible paths are formally weakly minimal and analysis of the proof of the Theorem 3.1 shows, that the theorem is valid for the rigid paths as well; in addition one can take $\hat{\psi}_{0}=0$. This leads to

Proposition 3.2. - If an admissible path $(\hat{u}(\cdot), \hat{q}(\cdot))$ is rigid on $[0, T]$, then $(\hat{u}(\cdot), \hat{q}(\cdot), T)$ is an abnormal geodesic path.

Remark. - As it is known [15], admissible paths (without or with pregiven end-points) of a completely nonholonomic vector distribution $\mathcal{D}$ are dense in metric of $C^{0}$ in the space of all paths on $M$ (correspondingly without or with pregiven end-points). Therefore an admissible path is never isolated in the metric of $C^{0}$. Therefore strong ( $=C^{0}$-local) minimality for sub-Riemannian geodesics need different treatment. The results on strong minimality of abnormal sub-Riemannian geodesics for 2-dimensional vector distributions are to be found in [26].

To finish with the 1st-order condition given by the Theorem 3.1 let us note that in the abnormal case the Hamiltonian (3.6) degenerates into an "abnormal" Hamiltonian

$$
H=\psi \cdot G(q) u .
$$

If we denote by $V_{\hat{u}(\tau)}^{\perp}$ the orthogonal complement to the vector $\hat{u}(\tau)$ in $R^{r}$, then the stationarity condition (3.7) for an abnormal geodesic takes form

$$
\hat{\psi}(\tau) \cdot G(\hat{q}(\tau)) v=0, \quad \forall v \in V_{\hat{u}(\tau)}^{\perp}, \quad \forall \tau \in[0, T],
$$

and (3.8) becomes:

$$
H(\hat{u}(\tau), \hat{q}(\tau), \hat{\psi}(\tau))=\hat{\psi}(\tau) \cdot G(\hat{q}(\tau)) \hat{u}(\tau)=0 .
$$

Vol. $13, \mathrm{n}^{\circ}$ 6-1996. 
Together with (3.10) it implies orthogonality of $\hat{\psi}(\tau)$ to the distribution $\mathcal{D}$ at every point $\hat{q}(\tau)$ :

$$
\hat{\psi}(\tau) \cdot G(\hat{q}(\tau)) v=0, \quad \forall v \in R^{r}, \quad \forall \tau \in[0, T] .
$$

\section{NECESSARY/SUFFICIENT CONDITIONS FOR RIGIDITY OF ABNORMAL SUB-RIEMANNIAN GEODESICS}

In the previous Section we have reduced the problem of finding minimal admissible ( $=$ tangent to the distribution $\mathcal{D}$ ) path between given points $q^{0}$ and $q^{1}$, to the Lagrange problem (3.1)-(3.3). We have formulated 1st-order necessary minimality condition saying that the solutions of this problem should be sought among geodesic paths. We have singled out the class of abnormal geodesics and defined what rigidity is. In this Section we are going to introduce 2 nd variation and set 2 nd-order necessary/sufficient conditions for rigidity of abnormal geodesic paths.

Let us start with definitions of first and second variations along an abnormal geodesic $(\hat{u}(\cdot), \hat{q}(\cdot), \hat{\psi}(\cdot), T)$. Everywhere in this Section we assume, that $\hat{u}(\cdot)$ is continuous (from the left) at $T$. We choose $\bar{T}>T$ and put $\hat{u}(t)=\hat{u}(T)$ on $[T, \vec{T}]$. Let us introduce a (time $\times$ input $) /$ state mapping $F: R \times L_{\infty}^{r}[0, \bar{T}] \longrightarrow M$, which maps a pair $(t, u(\cdot))$ into the point $q(t)$ of the trajectory $q(\cdot)$ of the system $\dot{q}=G(q) u(\tau), q(0)=q^{0}$. Obviously, $F(t, \hat{u}(\cdot))=\hat{q}(\cdot)$ and $F(T, \hat{u}(\cdot))=\hat{q}(T)=q^{1}$. We put

$\ell(t, u(\cdot))=\int_{0}^{t}\langle G(q(\tau)) u(\tau), G(q(\tau)) u(\tau)\rangle^{1 / 2} d \tau ; \ell: R \times L_{\infty}^{r}[0, \bar{T}] \rightarrow R$.

A well known fact is that for $(T, \hat{u}(\cdot)) \in R \times L_{\infty}^{r}$ to be a minimizer for the Lagrange problem (3.1)-(3.3) it must be critical point of the mapping $(\ell, F)$. Indeed otherwise in virtue of the Implicit Function Theorem the system of equations

$$
\ell(t, u(\cdot))=\ell(T, \hat{u}(\cdot))-\epsilon, \quad F(t, u(\cdot))=q^{1},
$$

is locally (in a neighborhood of $(T, \hat{u}(\cdot)))$ solvable for any sufficiently small $\epsilon>0$, and hence $q^{0}$ and $q^{1}$ can be connected by an admissible path of length $\ell(T, \hat{u}(\cdot))-\epsilon<\ell(T, \hat{u}(\cdot))$. If a pair $(T, \hat{u}(\cdot))$ is critical point for the mapping $(\ell, F)$, i.e. the differential $\left.\left(\ell^{\prime}, F^{\prime}\right)\right|_{(T, \hat{u}(\cdot))}: R \times L_{\infty}^{r} \rightarrow R \times \mathcal{T}_{q^{1}} M$ is nonsurjective, then there exists a pair $\left(\hat{\psi}_{0}, \hat{\psi}_{T}\right) \in R \times \mathcal{T}_{q^{1}}^{*} M$, which annihilates the image of $\left.\left(\ell^{\prime}, F^{\prime}\right)\right|_{(T, \hat{u}(\cdot))}$ :

$$
\hat{\psi}_{0} \ell^{\prime}+\hat{\psi}_{T} F^{\prime} \equiv 0 .
$$


This equality is equivalent to the statement of the Theorem 3.1 with $\hat{\psi}_{T}$ being the end-point value $\hat{\psi}(T)$ for the solution of the adjoint equation (3.5). If $\hat{\psi}_{0}=0$, then the functional $\ell$ does not enter both (4.1) and the Theorem 3.1. In this case the pair $(T, \hat{u}(\cdot))$ enters an abnormal geodesic $(\hat{u}(\cdot), \hat{q}(\cdot), \hat{\psi}(\cdot), T)$ or, equivalently, is critical point of the mapping $F$.

To study abnormal geodesics (= critical points of $F$ ) we have to invoke (first terms of) Taylor expansion for $F(t, u(\cdot))$. Let us present $F(t, u(\cdot))$ as chronological exponential (see Section 2 for the notation):

$$
F(t, u(\cdot))=q^{0} \circ \overrightarrow{\exp } \int_{0}^{t} G u(\tau) d \tau .
$$

Putting $u(\tau)=\hat{u}(\tau)+v(\tau)$ and using the variational formula (2.4) we obtain

$$
\begin{aligned}
F(t, u(\cdot)) & =q^{0} \circ \overrightarrow{\exp } \int_{0}^{t} G(\hat{u}(\tau)+v(\tau)) d \tau \\
& =q^{0} \circ \overrightarrow{\exp } \int_{0}^{t} G \hat{u}(\tau) d \tau \circ \overrightarrow{\exp } \int_{0}^{t} Y_{t, \tau} v(\tau) d \tau
\end{aligned}
$$

where

$$
Y_{t, \tau} v=\operatorname{Ad} \overrightarrow{\exp } \int_{t}^{\tau} G \hat{u}(\xi) d \xi G v
$$

From the formula $(2.5)$ it follows that

$$
d Y_{t, \tau} / d t=-\operatorname{ad} \hat{f}_{t} Y_{t, \tau}
$$

Putting

$$
Y_{\tau} v=Y_{T, \tau} v=\operatorname{Ad} \overrightarrow{\exp } \int_{T}^{\tau} G \hat{u}(\xi) d \xi G v
$$

we compute (compare with [7]) the first differential of $F$ at the point $(T, \hat{u}(\cdot))$ :

$$
\left.F^{\prime}\right|_{(T, \hat{u}(\cdot))}(\delta \theta, u(\cdot))=G\left(q^{1}\right) \hat{u}(T) \delta \theta+\int_{0}^{T} Y_{\tau}\left(q^{1}\right) u(\tau) d \tau, u(\tau) \in V_{\hat{u}(\tau)}^{\perp} .
$$

If a pair $(T, \hat{u}(\cdot))$ is critical point of $F$, then $\left.\operatorname{Im} F^{\prime}\right|_{(T, \hat{u}(\cdot))} \neq \mathcal{T}_{q^{1}} M$, and there exists a nonzero covector $\hat{\psi}_{T} \in \mathcal{T}_{q^{1}}^{*} M$, which annihilates $\left.\operatorname{Im} F^{\prime}\right|_{(T, \hat{u}(\cdot))}$. This implies

$$
\hat{\psi}_{T} \cdot G\left(q^{1}\right) \hat{u}(T)=0
$$

Vol. 13, n 6-1996. 
and

$$
\hat{\psi}_{T} \cdot \int_{0}^{T} Y_{\tau}\left(q^{1}\right) u(\tau) d \tau=0
$$

for all $u(\cdot) \in L_{\infty}^{r}[0, T]$ such that $u(\tau) \in V_{\hat{u}(\tau)}^{\perp}$. In virtue of DuboisRaymond Lemma the last equality implies:

$$
\hat{\psi}_{T} \cdot Y_{\tau}\left(q^{1}\right) v=0 \quad \forall v \in V_{\hat{u}(\tau)}^{\perp}, \text { for almost all } \tau \in[0, T] .
$$

These conditions are equivalent to the conditions (3.10)-(3.11) of the Theorem 3.1 with the "abnormal" Hamiltonian (3.9). Namely if we take the solution of the adjoint equation (3.5) with the end-point value $\hat{\psi}(T)=\hat{\psi}_{T}$, then the condition (4.7) is equivalent to the stationarity condition (3.7) and (4.6) implies, that the Hamiltonian $H=\psi G u$, being constant along $(\hat{u}(\cdot), \hat{q}(\cdot), \hat{\psi}(\cdot))$, vanishes. The corank of abnormal geodesic path $(\hat{u}(\cdot), \hat{q}(\cdot), T)$ coincides with the corank of $\left.F^{\prime}\right|_{(T, \hat{u}(\cdot))} \cdot$

Definition 4.1. - The first differential $\left.F^{\prime}\right|_{(T, \hat{u}(\cdot))}: R \times L_{\infty}^{r} \rightarrow \mathcal{T}_{q^{1}} M$, at a critical point $(T, \hat{u}(\cdot))$ is called first variation along abnormal geodesic path $(\hat{u}(\cdot), \hat{q}(\cdot), T)$. It is calculated according to the formula (4.5).

Now we introduce second variation along an abnormal geodesic $(\hat{u}(\cdot), \hat{q}(\cdot), \hat{\psi}(\cdot), T)$. It is Hessian, or quadratic differential of $F$, at the critical point $(T, \hat{u}(\cdot)) \in R \times L_{\infty}^{r}$ (see [10]). Choosing a function $\chi: M \longrightarrow R$, such that $\left.d \chi\right|_{q^{1}}=\hat{\psi}_{T}$, let us consider a function $\phi(t, u(\cdot))=$ $\chi(F(t, u(\cdot)))$. Since $\hat{\psi}_{T}$ annihilates $\left.\operatorname{Im} F^{\prime}\right|_{(T, \hat{u}(\cdot))}$, then $(T, \hat{u}(\cdot))$ is critical point for this function.

Let us compute the quadratic term of Taylor expansion for $\phi(t, u(\cdot))$ at $(T, \hat{u}(\cdot))$. Appealing to the Volterra expansion (2.2) for right cronolological exponential, we derive

$$
\begin{aligned}
& \left.\phi^{\prime \prime}\right|_{(T, \hat{u}(\cdot))}(\delta \theta, u(\cdot))=\left(\left(\int_{0}^{T} \int_{0}^{\tau} Y_{\xi} u(\xi) d \xi \circ Y_{\tau}(u(\tau) d \tau\right.\right. \\
& \left.-\int_{0}^{T}[G \hat{u}(T)), Y_{\tau}^{1} u(\tau)\right] d \tau \\
& \left.\left.\quad+(G \hat{u}(T)) \circ(G \hat{u}(T)) \frac{\delta \theta^{2}}{2}+(G \hat{u}(T)) \delta \theta \circ \int_{0}^{T} Y_{\tau} u(\tau) d \tau\right) \chi\right)\left(q^{1}\right) .
\end{aligned}
$$

(When carrying the computation one should take into account the equalities (3.8), (4.3) and (4.7).) 
When restricting the quadratic form (4.8) to the kernel of $\left.F^{\prime}\right|_{(T, \hat{u}(\cdot))}$, we are able to subtract from (4.8) a vanishing value of

$$
\frac{1}{2}\left(\left(G \hat{u}(T) \delta \theta+\int_{0}^{T} Y_{\tau} u(\tau) d \tau\right) \circ\left(G \hat{u}(T) \delta \theta+\int_{0}^{T} Y_{\tau} u(\tau) d \tau\right) \chi\right)\left(q^{1}\right)
$$

and transform (4.8) into

$$
\frac{1}{2}\left(\left(\int_{0}^{T}\left[\int_{0}^{\tau} Y_{\xi} u(\xi) d \xi, Y_{\tau} u(\tau)\right] d \tau\right)+\left[-G \hat{u}(T) \delta \theta, \int_{0}^{T} Y_{\tau} u(\tau) d \tau\right] \chi\right)\left(q^{1}\right)
$$

The last expression does not depend on choice of $\chi$ but only on $\hat{\psi}_{T}=\left.d \chi\right|_{q^{1}}$ and therefore we come to the

DEFINITION 4.2. - The quadratic form

$$
\begin{aligned}
& \left.2 F^{\prime \prime}\right|_{(T, \hat{u}(\cdot))}\left[\psi_{T}\right](\delta \theta, u(\cdot)) \\
& \quad=\hat{\psi}_{T} \cdot \int_{0}^{T}\left[-G \hat{u}(T) \delta \theta+\int_{0}^{\tau} Y_{\xi} u(\xi) d \xi, Y_{\tau} u(\tau)\right]\left(q^{1}\right) d \tau
\end{aligned}
$$

whose domain is subspace of $R \times L_{\infty}^{r}$ defined by the condition

$$
G\left(q^{1}\right) \hat{u}(T) \delta \theta+\int_{0}^{T} Y_{\tau}\left(q^{1}\right) u(\tau) d \tau=0, u(\tau) \in V_{\hat{u}(\tau)}^{\perp}, \tau \in[0, T],
$$

is called second variation along the abnormal geodesic $(\hat{u}(\cdot), \hat{q}(\cdot), \hat{\psi}(\cdot), T)$.

DEFINITION 4.3. - Morse index of abnormal geodesic is negative index of the quadratic form (4.9)-(4.10), i.e. maximal among the dimensions of the subspaces in its domain, on which the quadratic form is negative definite.

Definition 4.4. - Morse index of abnormal geodesic path $(\hat{u}(\cdot), \hat{q}(\cdot), T)$ is minimum of indices of the abnormal geodesics $(\hat{u}(\cdot), \hat{q}(\cdot), \hat{\psi}(\cdot), T)$, which correspond to this geodesic path, or minimum of indices of quadratic forms $\left.2 F^{\prime \prime}\right|_{(T, \hat{u}(\cdot))}\left[\hat{\psi}_{T}\right]$ for all possible $\left.\hat{\psi}_{T} \perp \operatorname{Im} F^{\prime}\right|_{(T, \hat{u}(\cdot))}$.

We now set 2 nd-order necessary rigidity condition for corank $k$ abnormal geodesics paths. It follows from general necessary condition for isolatedness of critical point of smooth mapping on critical level. Formulation and proof of the general condition (Theorem 9.1) as well as the proof of the following Theorem 4.1 are given in the Appendix (Section 9). Corresponding result for corank 1 case was established in [7], [8]. 
TheOREM 4.1 (Necessary Rigidity Condition for Abnormal Geodesics). For a corank $k$ abnormal geodesic path $(\hat{u}(\cdot), \hat{q}(\cdot), T)$ to be rigid its index should not exceed $k-1$. In particular index of a rigid corank 1 abnormal geodesic path must vanish.

Generally rigidity is stronger than weak minimality. But whenever all geodesics, which a geodesic path $(\hat{u}(\cdot), \hat{q}(\cdot), T)$ enters, are abnormal, then the conditions of the Theorem 4.1 are necessary for weak minimality of the path. It follows from the Propositions 9.4 and 9.3 (see Appendix).

Proposition 4.2 (Necessary Minimality Condition for Abnormal Geodesics). - Let $(\hat{u}(\cdot), \hat{q}(\cdot), T)$ be a corank $k$ abnormal geodesic path, such that all the corresponding geodesics are abnormal. Then for the geodesic path to be weakly minimal its index should not exceed $k-1$.

It follows from the Theorem 4.1, that finiteness of index is necessary for rigidity. Therefore we are going to invoke conditions which provide the finiteness for an abnormal geodesic $(\hat{u}(\cdot), \hat{q}(\cdot), \hat{\psi}(\cdot), T)$.

Denoting again by $V_{\hat{u}(\tau)}^{\perp}$ the orthogonal complement to $\hat{u}(\tau)$ in $R^{r}$ we introduce first of these conditions: for almost all $\tau \in[0, T]$

$$
\hat{\psi}(\tau) \cdot[G v, G w](\hat{q}(\tau))=0 \quad \forall v, w \in V_{\hat{u}(\tau)}^{\perp} .
$$

In different context it was introduced by B.S. Goh in [13] and we call it Goh necessary condition.

Differentiating the identity (3.10) w.r.t. $\tau$ one obtains for almost all $\tau \in[0, T]$

$$
0=\hat{\psi}(\tau) \cdot[G \hat{u}(\tau), G w](\hat{q}(\tau))=0 \quad \forall w \in V_{\hat{u}(\tau)}^{\perp} .
$$

and together with (4.11): for almost all $\tau \in[0, T]$

$$
\hat{\psi}(\tau) \cdot[G v, G w](\hat{q}(\tau))=0 \quad \forall v, w \in R^{r} .
$$

We will also refer to the last condition as to Goh condition. This condition together with (3.12) implies, that at every point $\hat{q}(\tau)$ of rigid abnormal geodesic $(\hat{u}(\cdot), \hat{q}(\cdot), \hat{\psi}(\cdot), T)$ the covector $\hat{\psi}(\tau)$ has to be orthogonal to $\mathcal{D}^{2}(\hat{q}(\tau))=[\mathcal{D}, \mathcal{D}](\hat{q}(\tau))$, spanned by the vector fields from $\mathcal{D}$ and their Lie brackets of the 2 nd order:

$$
\hat{\psi}(\tau) \cdot G v(\hat{q}(\tau))=0, \hat{\psi}(\tau) \cdot[G v, G w](\hat{q}(\tau))=0, \quad \forall v, w \in R^{r} .
$$

Another necessary condition, which is called (see [16], [4], [17]) Generalized Legendre Condition, is: for all $\tau \in[0, T]$

$$
\gamma_{\tau}(v, v)=\hat{\psi}(\tau) \cdot[[G \hat{u}(\tau), G v], G v](\hat{q}(\tau)) \geq 0
$$


Proofs can be found in in [4], [3], [1]; see also Appendix 2.

We summarize the aforesaid in following

Proposition 4.3 (Necessary Goh and Generalized Legendre Conditions). For an abnormal geodesic path $(\hat{u}(\cdot), \hat{q}(\cdot), T)$ to be rigid the Goh condition (4.13) and the Generalized Legendre Condition (4.15) have to hold for some abnormal geodesic $(\hat{u}(\cdot), \hat{q}(\cdot), \hat{\psi}(\cdot), T)$.

To set Jacobi-type conditions we need Strong Generalized Legendre Condition. It is (compare with (4.15)): for some $\beta>0$ and for all $\tau \in[0, T]$

$$
\gamma_{\tau}(v, v)=\hat{\psi}(\tau) \cdot[[G \hat{u}(\tau), G v], G v](\hat{q}(\tau)) \geq \beta\|v\|^{2}, \quad \forall v \in V_{\hat{u}(\tau)}^{\perp}
$$

This last condition, which together with (4.13) is sufficient for finiteness of Morse index of an abnormal geodesic, is not only essential for its rigidity but also provides smoothness and in some cases uniqueness of the geodesic.

THEOREM 4.4 (Regularity of Abnormal Geodesics). - Let Goh condition (4.11) and Strong Generalized Legendre Condition (4.16) hold along an abnormal geodesic $(\hat{u}(\cdot), \hat{q}(\cdot), \hat{\psi}(\cdot), T)$. Then the corresponding "control" $\hat{u}(\tau)$ and the trajectory $\hat{q}(\cdot)$ are smooth on $[0, T]$. If, in addition, the vector space $[\mathcal{D}, \mathcal{D}])\left(q^{0}\right)$ (correspondingly $[\mathcal{D}, \mathcal{D}]\left(q^{1}\right)$ ) has codimension 1 in $\mathcal{T}_{q^{0}} M$ (correspondingly in $\mathcal{T}_{q^{1}} M$ ), then no other abnormal geodesic path, starting at $q^{0}$ (correspondingly, finishing at $q^{1}$ ) may satisfy Goh condition (4.11) and Generalized Legendre Conditions (4.15).

Proof. - Differentiating (4.13) w.r.t. $\tau$, we obtain

$$
\hat{\psi}(\tau) \cdot[G \hat{u}(\tau),[G v, G w]](\hat{q}(\tau))=0, \quad \forall v, w \in R^{r}, \quad \forall \tau \in[0, T],
$$

and, in particular,

$$
\hat{\psi}(\tau) \cdot[G \hat{u}(\tau),[G \hat{u}(\tau), G v]](\hat{q}(\tau))=0, \quad \forall v \in R^{r}, \quad \forall \tau \in[0, T]
$$

Hence the points $(\hat{u}(\tau), \hat{q}(\tau), \hat{\psi}(\tau))$ of abnormal geodesic $(\hat{u}(\cdot), \hat{q}(\cdot), \hat{\psi}(\cdot))$ must lie in the subset of $S^{r-1} \times \mathcal{T}^{*} M$, defined by following system of relations:

$$
\begin{aligned}
& \Phi(u, q, \psi)=\psi \cdot[G u,[G u, G]](q)=0, \\
& \Omega(u, q, \psi)=\psi \cdot[[G u, G v], G v](q)>0, \quad \forall v \in V_{u}^{\perp} .
\end{aligned}
$$

Here $\Phi: R^{r} \times \mathcal{T}^{*} M \rightarrow\left(R^{r}\right)^{*}, \Omega: R^{r} \times \mathcal{T}^{*} M \rightarrow\left(R^{r}\right)^{*} \otimes\left(R^{r}\right)^{*}$ Vol. $13, n^{\circ}$ 6-1996. 
The differential of the mapping $\Phi$ w.r.t. $u$ at a point $(\hat{u}(\tau), \hat{q}(\tau), \hat{\psi}(\tau))$ is: $\Phi_{u}^{\prime} \Delta u=\hat{\psi}(\tau) \cdot[G \Delta u,[G \hat{u}(\tau), G]](\hat{q}(\tau))+\hat{\psi}(\tau) \cdot[G \hat{u}(\tau)[G \Delta u, G]](\hat{q}(\tau))$, or since in virtue of $(4.17)$ the last addend vanishes:

$$
\left.\Phi_{u}^{\prime}\right|_{(\hat{u}(\tau), \hat{q}(\tau), \hat{\psi}(\tau))} \Delta u=\hat{\psi}(\tau) \cdot[G \Delta u[G \hat{u}(\tau), G]](\hat{q}(\tau))
$$

Here $\Delta u \in V_{\hat{u}(\tau)}^{\perp} \simeq \mathcal{T}_{\hat{u}} S^{r-1}$, but if we substitute $\Delta u=\hat{u}(\tau)$ into (4.21), then in virtue of (4.18) $\left.\Phi_{u}^{\prime}\right|_{(\hat{u}(\tau), \hat{q}(\tau), \hat{\psi}(\tau))} \hat{u}(\tau)=0$. In virtue of (4.16) $\left.\Phi_{u}^{\prime}\right|_{(\hat{\hat{u}}(\tau), \hat{q}(\tau), \hat{\psi}(\tau))}$ is nonsingular on $V_{\hat{u}(\tau)}^{\perp}$ and hence the equation $\Phi(u, \hat{q}(\tau), \hat{\psi}(\tau))=0$ can be locally (in a small neighborhood of $(\hat{u}(\tau), \hat{q}(\tau), \hat{\psi}(\tau)))$ uniquely solved w.r.t. $u$, presenting $u$ as a smooth function $u=u(\hat{q}(\tau), \hat{\psi}(\tau))$.

In fact the solution of the system (4.19)-(4.20) is globally unique, even more, there is no other solution $\bar{u}(q, \psi)$ of the equation (4.19) such that $\hat{\psi}(\tau) \cdot[G v,[G \bar{u}, G v]](\hat{q}(\tau))$ is nonnegative quadratic form on $V_{\bar{u}}^{\perp}$. Indeed let us assume, that $\Phi(\hat{u}(\tau), \hat{q}(\tau), \hat{\psi}(\tau))=\Phi(\bar{u}, \hat{q}(\tau), \hat{\psi}(\tau))=0$. Then on the interval connecting $\hat{u}(\tau)$ with $\bar{u}$ there must be a point $u_{\mu}=\mu \hat{u}(\tau)+(1-\mu) \bar{u}(0<\mu<1)$ such that $\left.\Phi_{u}^{\prime}\right|_{\left(u_{\mu}, \hat{q}(\tau), \hat{\psi}(\tau)\right)}(\hat{u}(\tau)-\bar{u})=0$ or, since $\left.\Phi_{u}^{\prime}\right|_{\left(u_{\mu}, \hat{q}(\tau), \hat{\psi}(\tau)\right)}$ is linear w.r.t. $u_{\mu}$,

$$
\left(\left.\mu \Phi_{u}^{\prime}\right|_{(\hat{u}(\tau), \hat{q}(\tau), \hat{\psi}(\tau))}+\left.(1-\mu) \Phi_{u}^{\prime}\right|_{(\bar{u}, \hat{q}(\tau), \hat{\psi}(\tau))}\right)(\hat{u}(\tau)-\bar{u})=0 .
$$

The left-hand side of (4.22) belongs to $R^{r^{*}}$; applying it to the vector $(\hat{u}(\tau)-\bar{u}) \in R^{r}$ and taking into account, that $\left.\Phi_{u}^{\prime}\right|_{(\hat{u}(\tau), \hat{q}(\tau), \hat{\psi}(\tau))} \hat{u}(\tau)=$ $\left.\Phi_{u}^{\prime}\right|_{(\bar{u}, \hat{q}(\tau), \hat{\psi}(\tau))} \bar{u}=0$ we derive

$$
0=\left.\mu \Phi_{u}^{\prime}\right|_{(\hat{u}(\tau), \hat{q}(\tau), \hat{\psi}(\tau))}(\bar{u}, \bar{u})+\left.(1-\mu) \Phi_{u}^{\prime}\right|_{(\bar{u}, \hat{q}(\tau), \hat{\psi}(\tau))}(\hat{u}(\tau), \hat{u}(\tau))=0
$$

that may happen only if $\hat{u}(\tau)=\mp \bar{u}$. But for $\hat{u}(\tau)=-\bar{u}$, the quadratic form $\hat{\psi}(\tau) \cdot[[G \bar{u}, G v], G v](\hat{q}(\tau))$ is negative definite. Hence $\hat{u}(\tau)=\bar{u}$.

Thus we have established for every $\tau$ global uniqueness of the solution $\hat{u}(\tau)$ for the system of relations $\Phi(u, \hat{q}(\tau), \hat{\psi}(\tau))=0, \Omega(u, \hat{q}(\tau)$, $\hat{\psi}(\tau)) \geq 0$. Then the corresponding implicit function $u(q, \psi)$, which is defined by the system (4.19)-(4.20), is continuous and hence smooth w.r.t. $q, \psi$ and therefore $u(\hat{q}(\tau), \hat{\psi}(\tau))$ is smooth function of $\tau$.

Assume, that $\operatorname{codim}[\mathcal{D}, \mathcal{D}]\left(q^{0}\right)=1$. Then, as we will prove now, there is no other geodesic path, starting at $q^{0}$ and meeting Goh and Generalized Legendre Conditions (4.11) and (4.15). 
Assume on the contrary that there is another geodesic $(\bar{u}(\cdot), \bar{q}(\cdot), \bar{\psi}(\cdot), T)$ which starts at $q^{0}$ and meets the conditions (4.13) and (4.15). Then $\bar{\psi}(0)=k \hat{\psi}(0)$, or since the geodesic equations and the conditions (4.13) and (4.15) are homogeneous in $\hat{\psi}$, we may think, that $\bar{\psi}(0)=\hat{\psi}(0)$. We have already established existence of a unique smooth function $u(q, \psi)$ defined on some neighborhood $\mathcal{W}$ of the point $(\hat{q}(0), \hat{\psi}(0))$ such that: $\hat{u}(\tau)=u(\hat{q}(\tau), \hat{\psi}(\tau)), \bar{u}(\tau)=u(\bar{q}(\tau), \bar{\psi}(\tau))$. That means, that $(\hat{q}(\cdot), \hat{\psi}(\cdot))$ and $(\bar{q}(\cdot), \bar{\psi}(\cdot))$ are locally (in $\mathcal{W}$ ) solutions of the same Hamiltonian system with the same starting points. Hence they coincide in $\mathcal{W}$. Standard reasoning proves, that the set of those $t$, for which $(\hat{u}(\cdot), \hat{q}(\cdot), \hat{\psi}(\cdot))$ and $(\bar{q}(\cdot), \bar{q}(\cdot), \bar{\psi}(\cdot))$ coincide, is closed and open in $[0, T]$ and hence is $[0, T]$ itself. The same reasoning is applicable to the geodesic paths finishing at the point $q^{1}$.

Now we are going to set 2nd-order sufficient rigidity condition for abnormal geodesics. It involves the introduced above Goh and Generalized Strong Legendre Conditions, which provide for the second variation (4.9)(4.10) weak positive definiteness on some subspace of finite codimension in the domain (4.10) (see [1]). To put it in a strict way, let us note, that if Goh condition (4.11) holds, then the quadratic form (4.9)-(4.10) can be [1, Lemma 3.8] extended by continuity onto subspace of finite codimension in $R \oplus H_{-1}^{r}[0, T]$; the domain of the extension is determined by the condition (4.10). The notation $H_{-1}^{r}[0, T]$ stays for Sobolev space of order -1 , which is dual space to the space $H_{1}^{r}[0, T]$ of absolute-continuous functions with square integrable derivatives. The space $L_{2}^{r}[0, T]$ is densely embedded into $H_{-1}^{r}[0, T]$. For any function $u(\cdot) \in L_{2}^{r}[0, T] \subset H_{-1}^{r}[0, T]$, whose primitive is $v(\cdot)=\int_{0}^{\cdot} u(\tau) d \tau$, the $H_{-1}$-norm of $u(\cdot)$ can be defined as: $\|u(\cdot)\|_{-1}=\left(|v(T)|^{2}+\|v(\cdot)\|_{L_{2}}^{2}\right)^{1 / 2}$. There is a direct estimate

$$
\|u(\cdot)\|_{-1} \leq(T+\sqrt{T})\|u(\cdot)\|_{L_{2}} .
$$

Let us also note, that fast-oscillating functions have small $H_{-1}$-norms. Thus for a finite interval $[0, T]:\left\|\sin \delta^{-1} \tau\right\|_{-1}=O(\delta)$, for $\delta \rightarrow 0$, while $\left\|\sin \delta^{-1} \tau\right\|_{L_{2}}=T / 2+O(\delta)$, for $\delta \rightarrow 0$.

We define weak positive definiteness as positive definiteness w.r.t. the norm of $H_{-1}^{r}[0, T]$. We refer to [1] for the proof of the following

Proposition 4.5. - If Goh condition (4.11) and Generalized Strong Legendre Condition (4.16) both hold along abnormal geodesic, then the extension of the second variation (4.8) is weak positive definite on some subspace of finite codimension in its domain, i.e. the second variation admits on this subspace lower estimate:

$$
\left.2 F^{\prime \prime}\right|_{(T, \dot{u}(\cdot))}\left[\psi_{T}\right](\delta \theta, u) \geq c\left(\|u\|_{-1}^{2}+\delta \theta^{2}\right) .
$$


Corollary 4.6. - Under the conditions of the Proposition the second variation admits on some subspace of finite codimension in its domain a lower estimate

$$
\left.2 F^{\prime \prime}\right|_{(T, \hat{u}(\cdot))}\left[\psi_{T}\right](\delta \theta, u(\cdot)) \geq c\left(\|v(\cdot)\|_{L_{2}}^{2}+|v(T)|^{2}+\delta \theta^{2}\right),
$$

where $v(\cdot)=\int_{0} u(\tau) d \tau$.

Everywhere below we assume that Goh condition (4.11) and Generalized Strong Legendre Condition (4.16) hold along geodesics we deal with. This implies, that the negative indices of the second variation (4.9)-(4.10) and of its extension onto (subspace of) $R \times H_{-1}^{r}[0, T]$ are finite and coincide.

DEFINITION 4.5. - Nullity of an abnormal sub-Riemannian geodesic $(\hat{u}(\cdot), \hat{q}(\cdot), \hat{\psi}(\cdot), T)$ is the dimension of the kernel of the extended second variation (4.9)-(4.10) in $R \times H_{-1}^{r}[0, T]$.

It turns out, that in fact under the assumptions, we have made, the kernel "is almost contained in $R \times L_{\infty}^{r}$, namely it is contained in $R \times\left(L_{\infty}^{r} \oplus H_{-1}^{r}[0] \oplus H_{-1}^{r}[T]\right)$, where $H_{-1}^{r}[0]$ and $H_{-1}^{r}[T]$ consist of $R^{r}$-valued Dirac measures located at 0 and $T$ correspondingly. Following fact was established in [1].

Proposition 4.7. - Under Generalized Strong Legendre Condition (4.16) and Goh condition (4.13) the kernel of the extended second variation is contained in

$$
R \times\left(C^{\infty}\left((0, T), R^{r}\right) \oplus H_{-1}^{r}[0] \oplus H_{-1}^{r}[T]\right) .
$$

Now we are able to set Sufficient Rigidity Condition for abnormal geodesics.

THEOREM 4.8 (Sufficient Condition of Rigidity for Abnormal Geodesics). - If the second variation along abnormal geodesic $(\hat{u}(\cdot), \hat{q}(\cdot), \hat{\psi}(\cdot), T)$ is weakly positive definite, then the geodesic path $(\hat{u}(\cdot), \hat{q}(\cdot), T)$ is rigid, i.e. isolated up to a reparametrization in the topology of $W_{\infty}^{1}$ in the set of admissible paths, which connect $q^{0}$ and $q^{1}$.

Corollary 4.9. - If Goh condition (4.11) and Generalized Strong Legendre Condition (4.16) hold along abnormal geodesic $(\hat{u}(\cdot), \hat{q}(\cdot), \hat{\psi}(\cdot), T)$ and its Morse index and nullity both vanish, then the geodesic path $(\hat{u}(\cdot), \hat{q}(\cdot), T)$ is rigid.

The two results follow from general sufficient condition for isolatedness of critical points of smooth mappings on critical levels; formulation and proof of the general condition (Theorem 9.5) as well as the proof of the Theorem 4.8 are to be found in the Appendix (Section 9). 


\section{MORSE INDEX AND NULLITY OF ABNORMAL SUB-RIEMANNIAN GEODESICS}

In the previous Section we have formulated necessary (Theorem 4.1) and sufficient (Theorem 4.8) conditions for the rigidity of abnormal geodesics. The corresponding statements involve Morse index and nullity, and in this Section we are going to compute Morse index and nullity for an abnormal geodesic. The scheme of the computation is in many aspects similar to the one presented in [7], [8] for abnormal extremals of Lagrange Problem of Calculus of Variations. Refering to that paper for more details, we still provide a selfcontained exposition. The readers are referred to the Section 2 for notions and facts from symplectic geometry.

We start with the computation of the Morse index. To this purpose we introduce symplectic representation of the second variation (4.9)-(4.10) along abnormal geodesic $(\hat{u}(\cdot), \hat{q}(\cdot), \hat{\psi}(\cdot), T)$.

Let us put

$$
W=\operatorname{span}\left\{\left\{G\left(q^{1}\right) \hat{u}(T)\right\} \cup\left\{Y_{\tau}\left(q^{1}\right) v \mid \tau \in[0, T], v \in V_{\hat{u}(\tau)}^{\perp}\right\}\right\},
$$

where $Y_{\tau}$ is defined by (4.4). Evidently $W \subset \mathcal{T}_{q^{1}} M$ coincides with an image $\left.\operatorname{Im} F^{\prime}\right|_{(T, \hat{u}(\cdot))}$ of the first variation (4.5). It follows from (4.6)-(4.7), that $\hat{\psi}_{T}$ annihilates $W$, and codim $W=k$ is corank of the abnormal geodesic $(\hat{u}(\cdot), \hat{q}(\cdot), \hat{\psi}(\cdot), T)$.

Taking the space $\mathcal{E}_{W}$ of the vector fields, whose values at $q^{1}$ lie in $W$, let us consider a skewsymmetric bilinear form on $\mathcal{E}_{W}$ :

$$
\hat{\psi}_{T} \cdot\left[X, X^{\prime}\right]\left(q^{1}\right), \quad \forall X, X^{\prime} \in \mathcal{E}_{W}
$$

This form has kernel of finite codimension in $\mathcal{E}_{W}$, which is defined by equalities:

$$
X\left(q^{1}\right)=0 ; \hat{\psi}_{T} \cdot(\partial X / \partial \xi)\left(q^{1}\right)=0, \quad \forall \xi \in W
$$

Taking the quotient of $\mathcal{E}_{W}$ w.r.t. this kernel, one obtains on the finite-dimensional quotient space $\Sigma$ a (induced from (5.2)) nondegenerate skewsymmetric bilinear form $\sigma(\cdot, \cdot)$. This form defines symplectic structure on $\Sigma$. Direct calculation gives us $\operatorname{dim} \Sigma=2 \operatorname{dim} W=2(n-k)$. We denote by $\underline{X}$ the image of an $X \in \mathcal{E}_{W}$ under the canonical projection $\mathcal{E}_{W} \longrightarrow \Sigma$.

Choosing local coordinates $\left(x_{1}, \ldots x_{n}\right): \mathcal{O} \longrightarrow R^{n}$ on some neghborhood $\mathcal{O}$ of $q^{1}$ in $M$ in such a way that $x_{i}\left(q^{1}\right)=0,(i=1, \ldots n)$ and the subspace $W$ is defined by the equalities $x_{1}=\cdots=x_{k}=0$, while 
$\hat{\psi}_{T}=\left(\psi_{1}, \ldots, \psi_{k}, 0, \ldots 0\right)$, we may represent the canonical projection $X \rightarrow \underline{X}$ as:

$$
\begin{aligned}
X= & \sum_{i=1}^{n} X_{i}(x) \partial / \partial x_{i} \mapsto \underline{X} \\
= & \left(X_{k+1}(0), \ldots X_{n}(0)\right. \\
& \left.\partial\left(\sum_{i=1}^{k} \psi_{i} X_{i}\right) /\left.\partial x_{k+1}\right|_{0}, \ldots \partial\left(\sum_{i=1}^{k} \psi_{i} X_{i}\right) /\left.\partial x_{n}\right|_{0}\right)
\end{aligned}
$$

The symplectic form $\sigma(\underline{X}, \underline{Y})$ can be then represented as:

$$
\sigma(\underline{X}, \underline{Y})=\sum_{j=k+1}^{n}\left(X_{j}(0) \partial\left(\sum_{i=1}^{k} \psi_{i} Y_{i}\right) /\left.\partial x_{j}\right|_{0}-Y_{j}(0) \partial\left(\sum_{i=1}^{k} \psi_{i} X_{i}\right) /\left.\partial x_{j}\right|_{0}\right) .
$$

Let us denote by $\Pi$ the image under the canonical projection of the space of those vector fields, which vanish at $q^{1}$. Since the Lie bracket of two vanishing at $q^{1}$ vector fields also vanish at $q^{1}$, then $\Pi$ is Lagrangian plane.

Instead of notations $\underline{Y}_{\tau}$ and $G \hat{u}(T)$ for the images of the vector fields $Y_{\tau}$ and $G \hat{u}(T)$ under the canonical projection $\mathcal{E}_{W} \rightarrow \Sigma$ we use below $\Upsilon_{\tau}$ and $\hat{g}$ correspondingly. According to the introduced above definitions of $\sigma(\cdot, \cdot)$ and $\Pi$, we may represent the second variation (4.9)-(4.10) as:

$$
\left.2 F^{\prime \prime}\right|_{\left[T, \hat{u}(\cdot), \psi_{T}\right]}(\delta \theta, u(\cdot))=\int_{0}^{T} \sigma\left(-\hat{g} \delta \theta+\int_{0}^{\tau} \Upsilon_{\xi} u(\xi) d \xi, \Upsilon_{\tau} u(\tau)\right) d \tau
$$

and its domain as:

$$
\left\{(\delta \theta, u(\cdot)) \in R \times L_{\infty}^{r} \mid \hat{g} \delta \theta+\int_{0}^{T} \Upsilon_{\tau} u(\tau) d \tau \in \Pi\right\} .
$$

Under new notations the Goh condition (4.13) and Strong Generalized Legendre Condition (4.16) take form:

$$
\sigma\left(\Upsilon_{\tau} u, \Upsilon_{\tau} v\right)=0, \quad \forall u, v \in \dot{R}^{r}, \quad \forall \tau \in[0, T]
$$

and

$$
\gamma_{\tau}(u, u)=\sigma\left(\dot{\Upsilon}_{\tau} u, \Upsilon_{\tau} u\right) \geq \beta|u|^{2}, \quad \forall u \in V_{\hat{u}(\tau)}^{\perp},
$$

correspondingly.

Now we will transform the formulae (5.4)-(5.5) for the second variation, representing it as a quadratic form in $\delta \theta$ and $v(\cdot)=\int_{0} u(\xi) d \xi$ instead of 
$\delta \theta, u(\cdot)$. To this end let us integrate (5.4) by parts in such a way, that $u(\cdot)$ is integrated:

$$
\begin{aligned}
& \left.2 F^{\prime \prime}\right|_{\left[T, \hat{u}(\cdot), \psi_{T}\right]}(\delta \theta, u(\cdot))=\int_{0}^{T} \sigma(-\hat{g} \delta \theta+\int_{0}^{\tau} \Upsilon_{\xi} \underbrace{u(\xi) d \xi}_{d v}, \Upsilon_{\tau} u(\tau)) d \tau \\
& \quad=\int_{0}^{T} \sigma\left(-\hat{g} \delta \theta+\Upsilon_{\tau} v(\tau), \Upsilon_{\tau} u(\tau)\right) d \tau \\
& \quad-\int_{0}^{T} \sigma(\int_{0}^{\tau} \dot{\Upsilon}_{\xi} v(\xi) d \xi, \Upsilon_{\tau} \underbrace{u(\tau)) d \tau}_{d v} \\
& \quad=\int_{0}^{T} \sigma\left(\dot{\Upsilon}_{\tau} v(\tau), \Upsilon_{\tau} v(\tau)\right) d \tau \\
& \quad+\int_{0}^{T} \sigma\left(\hat{g} \delta \theta+\Upsilon_{T} v(T)+\int_{0}^{\tau} \dot{\Upsilon}_{\xi} v(\xi) d \xi, \dot{\Upsilon}_{\tau} v(\tau)\right) d \tau
\end{aligned}
$$

When proceeding with this computation we took into account that

$$
\sigma\left(\Upsilon_{\tau} v(\tau), \Upsilon_{\tau} u(\tau)\right)=0, \quad \sigma\left(\hat{g}, \Upsilon_{T} v(T)\right)=\sigma(\hat{g}, \underline{G v(T)})=0
$$

in virtue of Goh condition (5.6).

The domain of the second variation is:

$$
\left\{(\delta \theta, v(T), v(\cdot)) \mid \hat{g} \delta \theta+\underline{G v(T)}-\int_{0}^{T} \dot{\Upsilon}_{\tau} v(\tau) d \tau \in \Pi\right\} .
$$

Let us put

$$
\Gamma=\operatorname{span}\left\{\underline{G v} \mid v \in R^{r}\right\}, \quad \Gamma_{\varrho}=\operatorname{span}\left\{\underline{G v} \mid v \in V_{\hat{u}(T)}^{\perp}\right\}\left(\Gamma_{\varrho} \subset \Gamma\right) .
$$

In virtue of Goh condition (5.6) $\sigma(\underline{G v}, \underline{G w})=\sigma(\Upsilon v, \Upsilon w)=0$ and therefore $\Gamma$ (and $\Gamma_{\varrho}$ ) is isotropic subspace of $\Sigma: \Gamma \subseteq \Gamma^{b}$.

Following [1] we introduce now Hamiltonian form of Jacobi equation for abnormal geodesics. Considering the defined by (5.7) positive definite quadratic form $\gamma_{\tau}(u, u)$ on $V_{\hat{u}(\tau)}^{\perp}$, let us put $\bar{\gamma}_{\tau}$ for the nonsingular selfadjoint operator $\bar{\gamma}_{\tau}: V_{\hat{u}(\tau)}^{\perp} \longrightarrow V_{\hat{u}(\tau)}^{\perp *}$, which corresponds to $\gamma_{\tau}$ : $\gamma_{\tau}(u, v)=\left\langle\bar{\gamma}_{\tau} u, v\right\rangle, \forall u, v \in V_{\hat{u}(\tau)}^{\perp}$. Taking an inverse operator $\bar{\gamma}_{\tau}^{-1}$ : $V_{\hat{u}(\tau)}^{\perp^{*}} \longrightarrow V_{\hat{u}(\tau)}^{\perp}$, we define a bilinear form $\gamma_{\tau}^{-1}$ on $V_{\hat{u}(\tau)}^{\perp^{*}}$ as $\gamma_{\tau}^{-1}\left(u^{*}, v^{*}\right)=$ $\left\langle\bar{\gamma}_{\tau}^{-1} u^{*}, v^{*}\right\rangle, \forall u^{*}, v^{*} \in V_{\vec{u}(\tau)}^{\perp^{*}}$. Obviously for any $x \in \Sigma$ the mapping 
$u \longrightarrow \sigma\left(\dot{\Upsilon}_{\tau}, x\right)$ defines a linear form on $V_{\hat{u}(\tau)}^{\perp}$, i.e. an element of $V_{\hat{u}(\tau)}^{\perp^{*}}$, which depends linearly on $x \in \Sigma$. This means, that the correspondence

$$
x \longrightarrow \frac{1}{2} \gamma_{\tau}^{-1}\left(\sigma\left(\dot{\Upsilon}_{\tau} \cdot x\right)\right)
$$

defines a quadratic form on $\Sigma$.

Treating this quadratic form as time-dependent Hamiltonian on $\Sigma$, one may consider on $\Sigma$ linear Hamiltonian system:

$$
\dot{x}=\dot{\Upsilon}_{\tau} \bar{\gamma}_{\tau}^{-1}\left(\sigma\left(\dot{\Upsilon}_{\tau}, x\right)\right)
$$

which we call Jacobi equation for abnormal geodesic $(\hat{u}(\cdot), \hat{q}(\cdot), \hat{\psi}(\cdot), T)$.

If for any $\tau \in[0, T]$ the vectors $u_{1}(\tau), \ldots u_{r-1}(\tau)$ form such basises in $V_{\hat{u}(\tau)}^{\perp}$, that $\gamma_{\tau}\left(u_{i}(\tau), u_{j}(\tau)\right)=\delta_{i j},(i, j=1, \ldots r-1)$, then the equation can be presented as

$$
\dot{x}=\sum_{i=1}^{r-1} \sigma\left(\dot{\Upsilon}_{\tau} u_{i}(\tau), x\right) \dot{\Upsilon}_{\tau} u_{i}(\tau)
$$

Since a Hamiltonian flow preserves symplectic structure of $\Sigma$, then the Jacobi equation transforms Lagrangian planes into Lagrangian ones. Therefore one may consider the Hamiltonian flow as a flow on Lagrangian Grassmanian $\mathcal{L}(\Sigma)$. It is generated by the following time-dependent Hamiltonian system on $\mathcal{L}(\Sigma)$ :

$$
\dot{\Lambda}=\left.\frac{1}{2} \gamma_{\tau}^{-1}\left(\sigma\left(\dot{\Upsilon}_{\tau} \cdot x\right)\right)\right|_{\Lambda}
$$

(see Section 2 for details).

Definition 5.1. - Jacobi curve $\tau \mapsto \Lambda_{\tau}(\tau \in[0, T])$ corresponding to the abnormal geodesic $(\hat{u}(\cdot), \hat{q}(\cdot), \hat{\psi}(\cdot), T)$ is the curve in Lagrangian Grassmanian $\mathcal{L}(\Sigma)$, which starts at $\Lambda_{0}=\Pi$, coincides for $\tau \in[0, T)$ with the starting at $\Pi$ trajectory of the Jacobi equation (5.11) and jumps at $\tau=T-0$ to $\Lambda_{T}=\Lambda_{T-0}^{\Gamma}=\Lambda_{T-0} \cap \Gamma^{b}+\Gamma$.

Basing on this definition we set

ThEOREM 5.1 (Index Theorem for Abnormal Geodesics). - Let the Jacobi curve $\tau \mapsto \Lambda_{\tau}$ correspond to abnormal geodesic $(\hat{u}(\cdot), \hat{q}(\cdot), \hat{\psi}(\cdot), T)$. Then for any subdivision $\eta_{s+1}=0=\eta_{0}<\eta_{1}<\cdots<\eta_{s}=T$ of $\tau \mapsto \Lambda_{\tau}$ into 
simple subarcs $\left.\Lambda\right|_{\left[\eta_{i}, \eta_{i+1}\right]},(i=0, \ldots s-1)$ Morse index of the abnormal geodesic is equal to

$$
\sum_{i=0}^{s} \operatorname{ind}_{\Pi}\left(\Lambda_{\eta_{i}}, \Lambda_{\eta_{i+1}}\right)-(n-k)
$$

where $k$ is corank of the abnormal geodesic path $(\hat{u}(\cdot), \hat{q}(\cdot), T)$.

Proof. - We give a sketch of the proof of this Theorem, referring for details to the performed in [7] computation of Morse index of an abnormal extremal for Lagrange Problem of Calculus of Variations.

Putting $\delta \theta=0$ in (5.4)-(5.5), we obtain a quadratic form which we call reduced second variation. This quadratic form is a Hessian of input/state mapping (see [6]) $u(\cdot) \mapsto F(T, u(\cdot))$. Its domain has codimension 1 or 0 in the domain of the second variation (5.4)-(5.5), hence its index is not larger and differs at most by 1 from the index of the second variation. It can be represented as

$$
\left.2 F_{\varrho}^{\prime \prime}\right|_{\left[T, \hat{u}(\cdot), \psi_{T}\right]}(0, u(\cdot))=\int_{0}^{T} \sigma\left(\int_{0}^{\tau} \Upsilon_{\xi} u(\xi) d \xi, \Upsilon_{\tau} u(\tau)\right) d \tau
$$

with the domain

$$
\left\{(0, u(\cdot)) \mid \int_{0}^{T} \Upsilon_{\tau} u(\tau) d \tau \in \Pi\right\}
$$

We define Jacobi curve for the reduced 2nd variation following [1].

Definition 5.2. - Jacobi curve $\tau \mapsto \Lambda_{\tau}^{e}(\tau \in[0, T])$ corresponding to the reduced second variation (5.13)-(5.14) is the curve in the Lagrangian Grassmanian $\mathcal{L}(\Sigma)$, which starts at $\Lambda_{0}^{\varrho}=\Pi$ coincides for $\tau \in[0, T)$ with the starting at $\Pi$ trajectory of the Jacobi equation (5.11) and jumps at $\tau=T-0$ to $\Lambda_{T}^{\varrho}=\left(\Lambda_{T}^{\varrho}\right)^{\Gamma_{e}}=\Lambda_{T}^{\varrho} \cap \Gamma_{\varrho}^{b}+\Gamma_{\varrho}$.

The following Proposition providing formula for the index of the reduced second variation (5.13)-(5.14) via Maslov indices of the Jacobi curve $\Lambda_{\tau}^{\varrho}$ is corollary of the Theorem 1 in [1].

Proposition 5.2 (Index of the Reduced Second Variation). - Let $\tau \mapsto$ $\Lambda_{\tau}^{\varrho}(0 \leq \tau \leq T)$, be Jacobi curve corresponding to the reduced second variation (5.13)-(5.14) along an abnormal geodesic $(\hat{u}(\cdot), \hat{q}(\cdot), \hat{\psi}(\cdot), T)$. Then for any subdivision $\zeta_{m+1}=0=\zeta_{0}<\zeta_{1}<\cdots<\zeta_{m}=T$ of Vol. $13, n^{\circ} 6-1996$ 
$\tau \rightarrow \Lambda_{\tau}^{\varrho}$ into simple subarcs $\left.\Lambda^{\varrho}\right|_{\left[\zeta_{i}, \zeta_{i+1}\right]},(i=0, \ldots m-1)$ the negative index of the reduced second variation (5.13)-(5.14) is equal to

$$
\sum_{i=0}^{m} \operatorname{ind}_{I I}\left(\Lambda_{\zeta_{i}}^{\varrho}, \Lambda_{\zeta_{i+1}}^{\varrho}\right)+\operatorname{dim} \cap_{\tau \in[0, T]} \Lambda_{\tau}^{\varrho}-(n-k)
$$

where $k$ is corank of the abnormal geodesic path $(\hat{u}(\cdot), \hat{q}(\cdot), T)$.

Starting from the formula (5.15) one is able to compute negative index of the second variation (5.4)-(5.5), by using following technical Lemma (see [1]).

Proposition 5.3. - Assume, that a quadratic form $Q(\cdot, \cdot)$ is defined on a Hilbert space and is positive definite on a subspace of finite codimension. Let $\mathcal{N}$ be a closed subspace of the Hilbert space, $Q_{\mathcal{N}}$ be the restriction of $Q$ on $\mathcal{N}$, and $\mathcal{N}_{Q}^{\frac{1}{Q}}$ be the Q-orthogonal complement to $\mathcal{N}: \mathcal{N}_{\bar{Q}} \frac{1}{}=\{y \mid \mathcal{B}(x, y)=0, \forall x \in \mathcal{N}\}$, where $\mathcal{B}$ is corresponding to $Q$ symmetric bilinear form. Then

ind $Q=$ ind $Q_{\mathcal{N}}+$ ind $\left.Q\right|_{\mathcal{N}_{Q}^{\perp}}+\operatorname{dim}\left(\mathcal{N} \cap \mathcal{N}_{Q}^{\perp}\right)-\operatorname{dim}(\mathcal{N} \cap \operatorname{ker} Q)$.

To apply the result in our case we take for the Hilbert space $\mathcal{H}$ set of the pairs $(\delta \theta, u(\cdot))$, which meet the condition (5.5), for the subspace $\mathcal{N}$ the set of pairs $(0, u(\cdot))$, which meet the condition (5.14), and for $Q$ the quadratic form (5.4). Evidently $\operatorname{codim} \mathcal{N} \leq 1$

Following this line the authors have already computed in [7], [8] index of abnormal extremal for Lagrange Problem of Calculus of Variations. We have established in $[7,8]$, that appearance of additional term $\hat{g} \delta \theta$ in the second variation (5.4)-(5.5) in comparison with the reduced second variation (5.13)-(5.14) leads to a change of the final "jump" of the Jacobi curve, which becomes $\Lambda_{T}=\left(\Lambda_{T-0}^{\Gamma_{e}}\right)^{\hat{g}}=\Lambda_{T-0}^{\Gamma_{Q}} \cap \hat{g}^{b}+\operatorname{span}\{\hat{g}\}$. Since $\hat{g} \in \Gamma_{\varrho}^{b}$, then $\Gamma_{e}^{\hat{g}}=\Gamma_{e}+\operatorname{span}\{\hat{g}\}=\Gamma$ and also $\left(\Lambda_{T-0}^{\Gamma_{e}}\right)^{\hat{g}}=\Lambda_{T-0}^{\Gamma_{-0}}$. Therefore we come to the formula (5.12) (completing the proof of the Theorem 5.1).

Now we set Nullity Theorem for abnormal geodesics. Its proof is similar to the given in [7] proof of Nullity Theorem for abnormal extremals of Lagrange problem.

Theorem 5.4 (Nullity Theorem for Abnormal Geodesics). - Let $\tau \longrightarrow \Lambda_{\tau}$ be the Jacobi curve in Lagrangian Grassmanian $\mathcal{L}(\Sigma)$, which corresponds to an abnormal geodesic $(\hat{u}(\cdot), \hat{q}(\cdot), \hat{\psi}(\cdot), T)$. Then nullity of the abnormal geodesic, i.e. the dimension of the kernel of the second variation (5.4)-(5.5), is equal to $\operatorname{dim}\left(\Lambda_{T} \cap \Pi\right)$. 
What follows is corollary of the Theorems 5.1 and 5.4 (compare with the Corollary 5.5 in [7], Theorem 5 in [8]).

Theorem 5.5 (Local Rigidity Condition for Abnormal Geodesics). Let an abnormal geodesic $(\hat{u}(\cdot), \hat{q}(\cdot), \hat{\psi}(\cdot), T)$ meet Goh condition (4.11) and Strong Generalized Legendre Condition (4.16). Then for any small enough $\bar{t}>0$ the restrictions $\left(\left.\hat{u}(\cdot)\right|_{[0, \bar{t}]},\left.\hat{q}(\cdot)\right|_{[0, t]}, \bar{t}\right)$ of the geodesic path $(\hat{u}(\cdot), \hat{q}(\cdot), T)$ on $[0, \bar{t}]$ are rigid.

Proof. - Let us note firstly, that corank of the restrictions $\left(\left.\hat{u}(\cdot)\right|_{[0, \bar{t}},\left.\hat{q}(\cdot)\right|_{[0, t]}, \bar{t}\right)$ is integer-valued non decreasing function of $t$, and hence for small enough $t>0$ all the restrictions have the same corank $k>0$.

We are going to prove, that both index and nullity along any restriction $\left(\left.\hat{u}(\cdot)\right|_{[0, t]},\left.\hat{q}(\cdot)\right|_{[0, t]},\left.\hat{\psi}(\cdot)\right|_{[0, t]}, t\right)$ vanish and then apply the Corollary 4.9.

To compute the index of the restriction $\left(\left.\hat{u}(\cdot)\right|_{[0, t]},\left.\hat{q}(\cdot)\right|_{[0, t]},\left.\hat{\psi}(\cdot)\right|_{[0, t]}, t\right)$ let us consider corresponding nondecreasing Jacobi curve $\left.\Lambda_{\tau}\right|_{[0, t]}$, in Lagrangian Grassmanian. Since $\Gamma \cap \Pi=0$ then $\Gamma \cap \Lambda_{\tau}=0$ for any small enough $\tau>0$ and therefore $\operatorname{dim}\left(\Lambda_{\tau}^{\Gamma} \cap \Lambda_{\tau}\right)=$ const for small $\tau>0$. Then according to the Lemma 2.2 there exist $t>0$ and a Lagrangian plane $\Delta$ such that for any $\tau \in[0, t] \Lambda_{\tau}$ can be connected with $\Lambda_{\tau}^{\Gamma}$ by a simple nondecreasing curve $\Lambda_{\tau}(s), 0 \leq s \leq 1$ such that $\Lambda_{\tau}(s) \cap \Delta=0, \forall s \in[0,1]$. Then the concatenation of the curve $\left.\Lambda\right|_{[0, t]}$ with the corresponding curve $\Lambda_{t}(s)$ is also simple and evidently nondecreasing. According to the Proposition 5.2 and Theorem 5.1 index of the (having corank 1) restriction $\left(\left.\hat{u}(\cdot)\right|_{[0, t]},\left.\hat{q}(\cdot)\right|_{[0, t]},\left.\hat{\psi}(\cdot)\right|_{[0, t]}, t\right)$ equals

$$
\operatorname{ind}_{\Pi}\left(\Pi, \Lambda_{t}\right)+\operatorname{ind}_{\Pi}\left(\Lambda_{t}, \Lambda_{t}^{\Gamma}\right)+\operatorname{ind}_{\Pi}\left(\Lambda_{t}^{\Gamma}, \Pi\right)-(n-1),
$$

where $\Lambda_{t}^{\Gamma}=\Lambda_{t} \cap \Gamma^{b}+\Gamma$. According to the Lemma $2.1 \operatorname{ind}_{\Pi}\left(\Pi, \Lambda_{t}\right)+$ $\operatorname{ind}_{\Pi}\left(\Lambda_{t}, \Lambda_{t}^{\Gamma}\right)=\operatorname{ind}_{\Pi}\left(\Pi, \Lambda_{t}^{\Gamma}\right)$ for all small enough $t>0$ and we obtain for the Morse index the expression:

$$
\begin{aligned}
& \operatorname{ind}_{\Pi}\left(\Pi, \Lambda_{t}^{\Gamma}\right)+\operatorname{ind}_{\Pi}\left(\Lambda_{t}^{\Gamma}, \Pi\right)-(n-1) \\
& \quad=2 \frac{1}{2}\left(n-1-\operatorname{dim}\left(\Lambda_{t}^{\Gamma} \cap \Pi\right)\right)-(n-1)=-\operatorname{dim}\left(\Lambda_{t}^{\Gamma} \cap \Pi\right) \leq 0 .
\end{aligned}
$$

Being nonnegative this Morse index must vanish. That implies also $\operatorname{dim}\left(\Lambda_{t}^{\Gamma} \cap \Pi\right)=0$, i.e. in virtue of the Theorem 5.4 nullity of the restriction $\left(\left.\hat{u}(\cdot)\right|_{[0, t]},\left.\hat{q}(\cdot)\right|_{[0, t]},\left.\hat{\psi}(\cdot)\right|_{[0, t]}, t\right)$ also vanishes. 


\section{VECTOR DISTRIBUTIONS EXHIBITING RIGIDITY PHENOMENON}

We are going to describe some class of vector distributions, for which rigid abnormal geodesic paths do exist. We will consider germs of smooth $r$-dimensional vector distributions in $R^{n}$. It turns out that some conditions on growth vectors of the distributions provide existence of rigid geodesic paths.

TheOREM 6.1. - Let $n \geq 2 r, q^{0} \in M$. Then in the set of 2-jets at $q^{0}$ of vector distributions $\mathcal{D}$ satisfying the condition $\operatorname{dim} \mathcal{D}_{q}^{2} \equiv 2 r-1$ there is an open subset, such that for any distribution $\mathcal{D}$ with 2-jet lying in this subset, there exists a rigid admissible path starting at $q^{0}$.

Generic 2-dimensional distributions on $n$-dimensional manifold $M$ with $n \geq 4$ not only meet the conditions of the Theorem 6.1 , but possess stronger property.

THEOREM 6.2. - For any germ at a point $q^{0} \in M$ of 2-dimensional vector distribution $\mathcal{D}$, such that $\mathcal{D}_{q^{0}}^{3} \neq \mathcal{D}_{q^{0}}^{2}$, there exists rigid admissible path starting at $q^{0}$.

Proof of Theorem 6.1. - Let us assume that a distribution $\mathcal{D}$ meeting the conditions of the Theorem 6.1 is spanned by the vector fields $g^{1}, \ldots, g^{r}$, while

$$
\mathcal{D}^{2}=\operatorname{span}\left\{g^{1}, \ldots g^{r},\left[g^{1}, g^{2}\right], \ldots,\left[g^{1}, g^{r}\right]\right\} .
$$

Assume that for some $\psi^{0} \in \mathcal{T}_{q^{0}}^{*} M \backslash 0$, annihilating $\mathcal{D}^{2}$ the $((r-1) \times r)$-matrix

$$
A_{\psi}=\left(\begin{array}{ccc}
\psi\left[g^{1},\left[g^{1}, g^{2}\right]\right] & & \psi\left[g^{r},\left[g^{1}, g^{2}\right]\right] \\
\vdots & \cdots & \vdots \\
\psi\left[g^{1},\left[g^{1}, g^{r}\right]\right] & & \psi\left[g^{r},\left[g^{1}, g^{r}\right]\right]
\end{array}\right)
$$

has the maximal rank $(r-1)$. Then it holds also for a nonzero $\psi$ close to $\psi^{0}$ and there exists a smoothly depending on $\psi$ solution $u(\psi)=\left(u_{1}(\psi), \ldots u_{r}(\psi)\right) \in R^{r} \backslash 0$ of the systems $A_{\psi} u(\psi)=0$; without loss of generality we may assume $u\left(\psi^{0}\right)=(1,0 \ldots 0)$.

Assume in addition that the quadratic form

$$
L^{\psi^{0}}\left(v_{2}, \ldots, v_{r}\right)=\psi^{0}\left[\left[G u\left(\psi^{0}\right), G v\right], G v\right]\left(q^{0}\right)=\sum_{i, j=2}^{r} \psi^{0}\left[\left[g^{1}, g^{i}\right], g^{j}\right]\left(q^{0}\right) v_{i} v_{j}
$$

is positive definite. Hence for $\psi$ close to $\psi^{0}$ in $\mathcal{T}^{*} M$ the quadratic forms

$$
L^{\psi}(v)=\psi[[G u(\psi), G v], G v](q)
$$


are also positive definite on the orthogonal complements $V_{\psi}$ to $u(\psi)$ in $R^{r}$.

Any vector distribution meeting the conditions of the theorem and with 2 -jet belonging to a small enough neighborhood of the 2 -jet of $\mathcal{D}$ meets the above mentioned assumptions as well.

For any such vector distribution let us introduce a Hamiltonian $h(q, \psi)=$ $\sum_{i=1}^{r} u_{i}(\psi) \psi g^{i}(q)$. Then some subarc of the starting at $\left(q^{0}, \psi^{0}\right)$ trajectory $(\hat{q}(\cdot), \hat{\psi}(\cdot))$ of the corresponding Hamiltonian system is an abnormal geodesic, which meets Strong Generalized Legendre Condition. We need only to verify, that it also meets Goh condition, i.e. that $\psi \perp \mathcal{D}^{2}$ along the trajectory. In virtue of (6.1) it is enough to establish the equalities

$$
\psi g^{i} \equiv \psi\left[g^{1}, g^{i}\right] \equiv 0, \quad i=1, \ldots, r
$$

along the trajectory.

Let us put $h_{i}(t)=\psi g^{i}(\hat{q}(t), \hat{\psi}(t)), h_{1 i}(t)=\psi\left[g^{1}, g^{i}\right](\hat{q}(t), \hat{\psi}(t))(i=$ $1, \ldots, r)$ and compute $\dot{h}_{i}, \dot{h}_{1 i}$. Since $h=\sum_{j=1}^{r} u_{j}(\psi) h_{j}$, then

$$
\begin{aligned}
\dot{h}_{i} & =\left\{h, h_{i}\right\}(\hat{q}(t), \hat{\psi}(t))=\left\{\sum_{j=1}^{r} u_{j} h_{j}, h_{i}\right\}(\hat{q}(t), \hat{\psi}(t)) \\
& =\sum_{j=1}^{r} u_{j}(\psi)\left\{h_{j}, h_{i}\right\}(\hat{q}(t), \hat{\psi}(t))+\sum_{j=1}^{r}\left\{u_{j}, h_{i}\right\} h_{j}(\hat{q}(t), \hat{\psi}(t)) .
\end{aligned}
$$

In virtue of $(6.1)\left[g^{j}, g^{i}\right]$ lies in the linear span of vector fields $g^{1}, \ldots g^{r},\left[g^{1}, g^{2}\right], \ldots\left[g^{1}, g^{r}\right] ;$ hence

$$
\left\{h_{j}, h_{i}\right\}=\psi\left[g^{i}, g^{j}\right]=\sum_{k=2}^{r} \alpha_{k}(x) \psi\left[g^{1}, g^{k}\right]=\sum_{k=2}^{r} \alpha_{k} h_{1 k}
$$

and therefore

$$
\dot{h}_{i}(\hat{q}(t), \hat{\psi}(t))=\sum_{j=1}^{r}\left\{u_{j}, h_{i}\right\} h_{j}(\hat{q}(t), \hat{\psi}(t))+\sum_{j=1}^{r} v_{j}(\psi) h_{1 j}(\hat{q}(t), \hat{\psi}(t))
$$

Also

$$
\begin{gathered}
\dot{h_{1 i}}(\hat{q}(t), \hat{\psi}(t))=\left\{h, h_{1 i}\right\}(\hat{q}(t), \hat{\psi}(t))=\left\{\sum_{j=1}^{r} u_{j} h_{j}, h_{1 i}\right\}(\hat{q}(t), \hat{\psi}(t)) \\
=\sum_{j=1}^{r}\left(u_{j} \psi\left[g^{j},\left[g^{1}, g^{i}\right]\right](\hat{q}(t), \hat{\psi}(t))+\left\{u_{j}, h_{1 i}\right\} h_{i}\right)(\hat{q}(t), \hat{\psi}(t))
\end{gathered}
$$


666

$$
\begin{aligned}
& =A_{\psi} u(\psi)(\hat{q}(t), \hat{\psi}(t))+\sum_{j=1}^{r}\left\{u_{j}, h_{1 i}\right\} h_{i}(\hat{q}(t), \hat{\psi}(t)) \\
& =\sum_{j=1}^{r}\left\{u_{j}, h_{1 i}\right\} h_{i}(\hat{q}(t), \hat{\psi}(t)) .
\end{aligned}
$$

Therefore the functions $h_{i}(\hat{q}(t), \hat{\psi}(t)), \quad h_{1 i}(\hat{q}(t), \hat{\psi}(t)), \quad(i=1, \ldots r)$ satisfy the linear system of differential equations (6.2)-(6.3), and since

$$
h_{i}\left(q^{0}, \psi^{0}\right)=\psi^{0} g^{i}\left(q^{0}\right)=h_{1 i}\left(q^{0}, \psi^{0}\right)=\psi^{0}\left[g^{1}, g^{i}\right]\left(q^{0}\right)=0, \quad i=1, \ldots r
$$

then

$$
h_{i}(\hat{q}(t), \hat{\psi}(t))=h_{1 i}(\hat{q}(t), \hat{\psi}(t)) \equiv 0(i=1, \ldots, r) .
$$

We have established that the abnormal geodesic $(\hat{q}(\cdot), \hat{\psi}(\cdot))$ satisfies the conditions of the Theorem 5.5 and therefore is rigid.

To finish the proof of the Theorem 6.1 we only have to construct at least one $r$-dimensional distribution $\mathcal{D}$, with the growth vector and the basis meeting all the assumptions we have done.

Let $q^{0}=0_{R^{n}}$ and $R^{n}=\left\{(x, y, z) \mid x \in R^{r}, y \in R^{r}, z \in R^{n-2 r}\right\}$. Let us put

$$
\begin{aligned}
& g^{1}=\partial / \partial x_{1} ; g^{i}=\partial / \partial x_{i}+x_{1} \partial / \partial y_{i}+y_{i} \partial / \partial y_{1}, \quad i=2, \ldots, r-1 \\
& g^{r}=\partial / \partial x^{r}+x^{1} \partial / \partial y_{r}+y_{r} \partial / \partial y_{1}+\sum_{j=1}^{n-2 r} x_{1}^{j+1} \partial / \partial z_{j}
\end{aligned}
$$

The vector fields $g^{1}, \ldots, g^{r}$ span germ of $r$-dimensional distribution of full Lie rank. In addition:

$$
\begin{gathered}
{\left[g^{1}, g^{i}\right]=\partial / \partial y_{i}, i=2, \ldots, r-1} \\
{\left[g^{1}, g^{r}\right]=\partial / \partial y_{r}+\sum_{j=1}^{n-2 r}(j+1) x_{1}^{j} \partial / \partial z_{j}} \\
{\left[g^{i}, g^{j}\right]=0, i, j=2, \ldots r ; \quad\left[g^{1},\left[g^{1}, g^{i}\right]\right]=0, \quad i=2, \ldots, r-1}
\end{gathered}
$$

$\left[g^{1},\left[g^{1}, g^{r}\right]\right]$ is linear combination of $\partial / \partial z_{j}$, and $\left[\left[g^{1}, g^{i}\right], g^{j}\right]=\delta_{i j} \partial / \partial y_{1}$, where $\delta_{i j}$ is Kronecker symbol. All the above mentioned assumptions will hold if we choose $\psi^{0}$ such that

$$
\begin{aligned}
& \psi^{0} \partial / \partial x_{i}=\psi^{0} \partial / \partial z_{j}=0, \quad i, j=1, \ldots, r \\
& \psi^{0} \partial / \partial y_{i}=0, \quad i=2, \ldots, r ; \quad \psi^{0} \partial / \partial y_{1}=1
\end{aligned}
$$


Proof of Theorem 6.2. - One can always choose vector fields $f, g$, which span $\mathcal{D}$ and a covector $\psi^{0} \in \mathcal{T}_{q^{0}}^{*} M \backslash 0$, in such a way, that following conditions hold:

$$
\begin{aligned}
& \psi^{0} f\left(q^{0}\right)=\psi^{0} g\left(q^{0}\right)=\psi^{0}[f, g]\left(q^{0}\right)=\psi^{0}[f,[f, g]]\left(q^{0}\right)=0 \\
& \psi^{0}[g,[f, g]]\left(q^{0}\right)>0
\end{aligned}
$$

(it is enough to chose $\psi^{0} \perp \mathcal{D}^{2}, \psi^{0} \not \mathcal{D}^{3}$ and, when necessary, multiply $\psi^{0}$ by -1 ). The inequality (6.4) holds for all $\psi$ close to $\psi^{0}$ in $\mathcal{T}^{*} M$. Considering the equation

$$
\Phi(\psi, u)=\psi[f+g u,[f, g]](q)=0
$$

we note, that, since $\psi^{0}[g,[f, g]]\left(q^{0}\right)>0$ then locally the equation $\Phi(\psi, u)=0$ has smooth solution $u(\psi)=(\psi[f,[f, g]](q)) /(\psi[g,[f, g]](q))$; $u\left(\psi^{0}\right)=0$. As in the proof of the previous theorem some subarc of starting at $\left(q^{0}, \psi^{0}\right)$ trajectory of Hamiltonian system with the Hamiltonian $h=\psi(f+g u(\psi))$ is abnormal geodesic, which meets Goh and Strong Generalized Legendre Conditions and hence is rigid.

\section{ABNORMAL GEODESICS FOR 2-DIMENSIONAL VECTOR DISTRIBUTIONS: FEW MORE STEPS}

In this Section we deal with abnormal geodesics for 2-dimensional smooth vector distributions. For this case we are able to proceed further with the computation of Morse index and nullity and obtain elegant representation of the Jacobi equation and characterization of conjugate points.

Let us consider a 2 -dimensional distribution $\mathcal{D}$ on a $(n+2)$-dimensional manifold; let the vector fields $f, g \in \operatorname{Vect} M$ span $\mathcal{D}$. Assume that:

i) the vector fields

$$
f, g,[f, g], \ldots,(\operatorname{ad} f)^{n-1} g
$$

are linearly independent at every point of the domain we treat;

ii) $(\operatorname{ad} f)^{n} g$ can be presented as a linear combination with $C^{\infty}$-coefficients of these $n+1$ vector fields:

$$
(\operatorname{ad} f)^{n} g=\beta f+\sum_{i=0}^{n-1} \alpha^{i}(\operatorname{ad} f)^{i} g\left(\beta, \alpha^{i} \in C^{\infty}(M)\right) .
$$


Then the trajectories of the vector field $f$ are corank 1 abnormal geodesics for the distribution $\mathcal{D}$. Let us consider vector distribution (free $C^{\infty}(M)$ )module of vector fields)

$$
V=\operatorname{span}\left\{f, g,[f, g], \ldots(\operatorname{ad} f)^{n-1} g\right\},
$$

and assume, that:

iii) in the treated domain

$$
[[f, g] g]](q) \notin V(q) .
$$

Let $\psi$ be a 1 -form on the domain, defined by the conditions:

$$
\psi \perp V, \quad \psi[[f, g] g]=1 .
$$

We shall derive Jacobi equation for the abnormal geodesic, which corresponds to the vector field $f$. We denote by $\hat{q}(\cdot)=q^{0} \circ e^{t f}$ the starting at $q^{0}=\hat{q}(0)$ trajectory of $f ; \hat{q}(T)=q^{1}$. Following the approach of the Section 5 let us consider skewsymmetric bilinear form $\left(v_{1}, v_{2}\right) \mapsto \psi \cdot\left[v_{1}, v_{2}\right]\left(q^{1}\right), v_{1}, v_{2} \in V$. Taking quotient of $V$ w.r.t. the kernel of this form we obtain a $2(n+1)$-dimensional symplectic space $\Sigma^{\prime}$. We reduce the symplectic space considering the $(2 n+1)$-dimensional skeworthogonal complement to the canonical projection $\hat{f}$ of the vector field $f$ onto $\Sigma^{\prime}$ and then taking quotient of $\Sigma^{\prime}$ w.r.t. $\operatorname{span}\{\hat{f}\}$. The result is denoted further by $\Sigma$; it is $2 n$-dimensional symplectic space with skewscalar product denoted by $\sigma$. We again denote by $\underline{Y}$ the image of a vector field $Y \in V$ under the canonical projection $V \rightarrow \Sigma$.

We are going to introduce special coordinates in $\Sigma$ and to derive one more representation of the Jacobi equation (5.10).

Let us put for $i \geq 0$

$$
\begin{aligned}
g_{t} & =e^{(t-T) \operatorname{ad} f} g, \quad g_{t}^{i}=e^{(t-T) \operatorname{ad} f}(\operatorname{ad} f)^{i} g=\partial^{i} g_{t} / \partial t^{i}, \\
\gamma_{t}^{i} & =\hat{\psi} \cdot\left[g_{t}^{1}, g_{t}^{i}\right]\left(q^{1}\right)=\hat{\psi}(t)\left[[f, g],(\operatorname{ad} f)^{i} g\right](\hat{q}(t)) .
\end{aligned}
$$

Returning to the equality (7.1) we put $\alpha_{t}^{i}=\alpha^{i}(\hat{q}(t))(i=0, \ldots n-1)$, $\beta_{t}=\beta(\hat{q}(t))$, and derive from (7.1):

$$
g_{t}^{n}\left(q^{1}\right)=\beta_{t} f\left(q^{1}\right)+\sum_{i=0}^{n-1} \alpha_{t}^{i} g_{t}^{i}\left(q^{1}\right)
$$

LEMMA 7.1.

$$
\underline{g}_{t}^{n}=\sum_{i=0}^{n-1} \alpha_{t}^{i} \underline{g}_{t}^{i}
$$


Proof. - Chosing coordinates in $\Sigma$ as in (5.3) (with $k=1$ ) we only need to establish, that

$$
\partial\left(\psi \cdot g_{t}^{n}\right) /\left.\partial x\right|_{q^{1}}=\sum_{i=0}^{n-1} \alpha_{t}^{i} \partial\left(\psi \cdot g_{t}^{i}\right) /\left.\partial x\right|_{q^{1}}+\beta_{t} \partial(\psi \cdot f) /\left.\partial x\right|_{q^{1}}
$$

for local coordinates $x=\left(x_{1}, \ldots x_{n}\right)$ in the neighborhood of $q^{1} \in M$. But this follows directly from (7.1) and the equalities $\left.\left(\psi g_{t}^{i}\right)\right|_{q^{1}} \equiv 0$, $i=0, \ldots n-1$.

Let $\Pi$ be image under the canonical projection of the vector fields $Y$, which meet the condition $\psi \cdot[f, Y]\left(q^{1}\right)=0$ and vanish at $q^{1} ; \Pi$ is Lagrangian plane in $\Sigma$. It follows from (7.1)-(7.2), that $\Sigma=\Pi \oplus \operatorname{span}\left\{\underline{g}_{t}, t \in R\right\}$ and for any $\tau \in R$ the vectors $\underline{g}_{\tau}, \underline{g}_{\tau}^{1} \ldots \underline{g}_{\tau}^{n-1}$ form the basis of the subspace $\operatorname{span}\left\{\underline{g}_{t}, t \in R\right\}=\Delta$. Let us emphasize, that the subspace $\Delta$ is not Lagrangian and $\sigma$ defines a nondegenerate coupling between $\Pi$ and $\Delta$.

Representing a $x \in \Sigma$ as $x=z+\xi$, where $z \in \Delta, \xi \in \Pi$, we may write the Jacobi equation (see (5.10)) in these coordinates as

$$
\gamma_{t}^{0}(\dot{z}+\dot{\xi})=\sigma\left(\underline{g}_{t}^{1}, z+\xi\right) \underline{g}_{t}^{1}
$$

or

$$
\gamma_{t}^{0} \dot{z}=\sigma\left(\underline{g}_{t}^{1}, z\right) \underline{g}_{t}^{1}+\sigma\left(\underline{g}_{t}^{1}, \xi\right) \underline{g}_{t}^{1}, \dot{\xi}=0 .
$$

Evidently one of the solutions of this equation is: $z_{t} \equiv \underline{g}_{t}, \xi_{t}=0$.

We call $\bar{t}$ a conjugate point of multiplicity $k>0$, for the abnormal geodesic $\hat{q}(t)=q^{0} \circ e^{t f}$, if for the equation (7.3) the space of solutions, which satisfy boundary conditions

$$
z_{0}=0, z_{\bar{t}} \| \underline{g}_{\bar{t}}, \sigma\left(\underline{g}_{0}, \xi_{0}\right)=0
$$

is $k$-dimensional.

Let us put $\zeta_{t}=\sigma\left(\underline{g}_{t}, \xi_{0}\right)$ and present $z_{t}$ in the form: $z_{t}=\sum_{i=0}^{n-1} z_{t}^{i} \underline{g}_{t}^{i}$. Then the equation (7.3) can be transformed into the following system

$$
\left.\begin{array}{rl}
\gamma_{t}^{0}\left(\dot{z}^{1}+\alpha_{t}^{1} z^{n-1}\right) & =\sum_{j=2}^{n-1} \gamma_{t}^{j} z^{j}+\dot{\zeta}, z_{0}^{1}=0 \\
\dot{z}^{j}+\alpha_{t}^{j} z^{n-1} & =-z^{j-1}, z_{0}^{j}=0, j=2, \ldots n-1, \\
\zeta^{(n)} & =\sum_{i=0}^{n-1} \alpha_{t}^{i} \zeta^{(i)}, \zeta_{0}=0 .
\end{array}\right\}
$$

(the equation for $z^{0}$, which enters neither (7.4) nor (7.5), is ommitted). 
The multiplicity of a conjugate point is the dimension of the space of those solutions of the system (7.5), which satisfy the conditions

$$
z_{\bar{t}}^{i}=0, \quad i=1, \ldots, n-1
$$

Summarizing the aforesaid we set following

THEOREM 7.1. - Assume that for starting at $q^{0}$ trajectory $\hat{q}(t)=q^{0} \circ e^{t f}$ of 2-dimensional vector distribution on an $(n+2)$-dimensional manifold the set above conditions (i), (ii) and (iii) hold. Then:

1) $\hat{q}(t), t \in[0, T]$, is corank 1 abnormal geodesic path of the distribution;

2) it has finite number (may be zero) of conjugate points $\bar{t}_{i}$ and multiplicity of a conjugate point $\bar{t}$ is equal to the dimension of the space of solutions of the system (7.5), which satisfy the boundary conditions (7.6);

3) Morse index of the abnormal geodesic is equal to the sum of multiplicities of the conjugate points, which are located on $(0, T)$;

4) nullity of the abnormal geodesic is equal to the multiplicity of conjugate point at $T$ (vanishes if $T$ is not a conjugate point);

5) for the abnormal geodesic path to be rigid it is necessary, that $(0, T)$ does not contain conjugate points, and it is sufficient, that $(0, T]$ does not contain conjugate points.

Proof. - Statement 1) was established at the beginning of the Section, finiteness of the set of conjugate points and the statements 3 ), 4) follow from strong regularity (see [22]) of the abnormal geodesic $\hat{q}(\cdot)$; statement 5) follows from corank 1 variants of the Theorems 4.1 and 4.8 together with 3 ) and 4).

Now we treat in more detailed way the case $n=2$, i.e. 2-dimensional vector distributions on 4-dimensional manifolds. Here the vector field $f$, which meets the condition (7.1) exists and is unique for any 2-dimensional distribution of maximal growth; such vector distributions define so-called Engel structure on 4-dimensional manifolds (readers can find in [12] a detailed survey of this topic). For $n=2$ the system (7.5) takes form:

$$
\left.\begin{array}{c}
\dot{z}^{1}=-\alpha_{t}^{1} z^{1}+\frac{\dot{\zeta}}{\gamma_{t}^{0}}, \quad z_{0}^{1}=0, \\
\ddot{\zeta}=\alpha_{t}^{0} \zeta+\alpha_{t}^{1} \dot{\zeta}, \quad \zeta_{0}=0, \dot{\zeta}_{0}=1 .
\end{array}\right\}
$$

Besides

$$
\dot{\gamma}_{t}^{0}=d\left(\psi\left[g_{t}^{1}, g_{t}\right]\right) / d t=\psi\left[g_{t}^{2}, g_{t}\right]=\alpha_{t}^{1} \gamma_{t}^{0}, \quad \gamma_{0}^{0}=1
$$


Hence $\gamma_{t}^{0}=e^{\int_{0}^{t} \alpha_{\tau}^{1} d \tau}$, and therefore

$$
z_{t}^{1}=\int_{0}^{t} \frac{1}{\gamma_{\tau}^{0}} e^{-\int_{\tau}^{t} \alpha_{\theta}^{1} d \theta} \dot{\zeta}_{\tau} d \tau=\zeta_{t} e^{-\int_{0}^{t} \alpha_{\tau}^{1} d \tau}
$$

One sees, that $\bar{t}$ is a conjugate point if and only if $\zeta_{\bar{t}}=0$. Multiplicity of any conjugate point equals to 1 .

Therefore for the 2-dimensional case the following corollary of the Theorem 7.1 is valid.

COROLLARY 7.2. - For every 2-dimensional distribution of maximal growth on 4-dimensional manifold $M$ and for every point $q^{0} \in M$ there exists a corank 1 abnormal geodesic path $\hat{q}(t),(t \in[0, T])$ of the distribution starting at $q^{0}$. Morse index of the corresponding abnormal geodesic is equal to the number of located on $(0, T)$ zeros of the solution $\zeta(\cdot)$ of the equation (7.7). For the abnormal geodesic path to be rigid it is necessary (correspondingly, sufficient), that there are no zeros of $\zeta(\cdot)$ on $(0, T)$ (correspondingly, on $(0, T]$ ).

\section{RIGID TRAJECTORIES OF AFFINE CONTROL SYSTEMS}

In the Section we extend our approach onto the class of affine control systems

$$
\dot{q}=f(q)+\sum_{i=1}^{r} g^{i}(q) u_{i}, \quad q(0)=q^{0}
$$

and derive rigidity conditions for extremals of these systems.

Here the drift vector field $f(q)$ and the control vector fields $g^{i}(q), i=$ $1, \ldots r$, are $C^{\infty}$ on $M$ admissible controls $u(\cdot)=\left(u_{1}(\cdot), \ldots u_{r}(\cdot)\right) \in L_{\infty}^{r}$.

The material of this section relates to the results of $[7,8]$, where nonlinear Lagrange Problem of the Calculus of Variations was treated, and also with [11], which treated time-optimal problems for affine control systems.

We start with definition of rigidity for a given input $\hat{u}(t), t \in[0, T]$ of the affine control system $(8.1)$, We assume $\hat{u}(\cdot)$ to be continuous at $T-0$. The extension of $\hat{u}(\cdot)$ from $[0, T]$ onto $[0, T+\delta]$ by the constant $\hat{u}(T)$ will be denoted also by $\hat{u}(\cdot)$. We assume that the starting at $q^{0}$ trajectory $\hat{q}(\cdot)$ of the system $(8.1)$ driven by the control $\hat{u}(\cdot)$ exists on $[0, T+\delta]$.

DEFINITION 8.1. - A control $\hat{u}(\cdot)$ and the corresponding trajectory $\hat{q}(\cdot)$ of the control system (8.1) are called rigid on $[0, T]$, if for some $\epsilon>0$ no one Vol. $13, \mathrm{n}^{\circ}$ 6-1996. 
(different from $\hat{u}(\cdot)$ ) control from $\epsilon$-neighborhood of $\hat{u}(\cdot)$ in $L_{\infty}^{r}$ can steer the system (8.1) from $q^{0}$ to $\hat{q}(T)$ in a time $T^{\prime} \in[T-\epsilon, T+\epsilon]$.

Following the approach of the Section 4 we consider for the system (8.1) a time $\times$ input/state mapping $F: R \times L_{\infty}^{r} \rightarrow M ; F$ maps a pair $(t, u(\cdot))$ consisting of time moment $t$ and an admissible control $u(\cdot)$ into the point $q(t)$ of the trajectory $q(\cdot)$ of the control system (8.1).

It turns out that for $(\hat{u}(\cdot), \hat{q}(\cdot), T)$ to be rigid, $(T, \hat{u}(\cdot))$ has to be critical point of $F$. That means $\left.\operatorname{Im} F^{\prime}\right|_{(T, \hat{u}(\cdot))} \neq \mathcal{T}_{\hat{q}(T)} M$, and there exists nonzero $\hat{\psi}_{T} \in \mathcal{T}_{\hat{q}(T)}^{*} M$ annihilating $\left.\operatorname{Im} F^{\prime}\right|_{(T, \hat{u}(\cdot))}$. For any such $\hat{\psi}_{T}$ one can transform the equality $\hat{\psi}_{T} F^{\prime} \equiv 0$ into Hamiltonian form of the EulerLagrange equation (compare with the Theorem 3.1), which is extremality condition for the path $(\hat{u}(\cdot), \hat{q}(\cdot))$. It is more convenient for us to set it this time as a Definition.

DEFINITION 8.2 (Extremality). - We call $(\hat{u}(\cdot), \hat{q}(\cdot))$ extremal pair for the affine control system (8.1) on $[0, T]$, if there exists an absolutely-continuous covector-function $\hat{\psi}(\tau)$ on $[0, T])$ such that the triple $(\hat{u}(\cdot), \hat{q}(\cdot), \hat{\psi}(\cdot))$ :

1) satisfies Hamiltonian system

$$
\begin{aligned}
& \dot{q}=\partial H / \partial \psi, \quad q(0)=q^{0}, \\
& \dot{\psi}=-\partial H / \partial q,
\end{aligned}
$$

with an "affine" Hamiltonian

$$
H_{f}(u, q, \psi)=\psi \cdot(f(q)+G(q) u)
$$

2) meets stationarity condition

$$
\left.\frac{\partial H_{f}}{\partial u}\right|_{(\hat{u}(\tau), \hat{q}(\tau), \hat{\psi}(\tau))}=\hat{\psi}(\tau) G(\hat{q}(\tau))=0, \text { for almost all } \tau \in[0, T],
$$

and "transversality condition"

$$
H_{f}(\hat{u}(\tau), \hat{q}(\tau), \hat{\psi}(\tau))=0, \text { for almost all } \tau \in[0, T]
$$

We call $\hat{u}(\cdot)$ extremal control and $\hat{q}(\cdot)$ extremal trajectory of the control system $(8.1)$ on $[0, T]$.

The quadruple $(\hat{u}(\cdot), \hat{q}(\cdot), \hat{\psi}(\cdot), T)$ is called extremal of the control system (8.1) on $[0, T]$.

Corank of an extremal path $(\hat{u}(\cdot), \hat{q}(\cdot), T)$ is the dimension of the space of extremals, it enters, or, equivalently, dimension of the space of those 
$\hat{\psi}(\cdot)$, which together with $(\hat{u}(\cdot), \hat{q}(\cdot), T)$ satisfy the Definition 8.2 , or, equivalently, corank $\left.F^{\prime}\right|_{(T, \hat{u}(\cdot))}$.

It follows from the Implicit Function Theorem, that extremality is necessary for rigidity.

Proposition 8.1. - A rigid path $(\hat{u}(\cdot), \hat{q}(\cdot), T)$ of the control system (8.1) must be extremal pair of the system.

Let us put $\hat{f}_{\tau}(q)=f(q)+\sum_{i=1}^{r} g^{i}(q) u_{i}(\tau)$. To derive 2nd-order rigidity conditions we have to involve again Goh and Generalized Legendre Conditions along extremal. They are (compare with (4.11) and (4.15)):

$$
\hat{\psi}(\tau) \cdot[G v, G w](\hat{q}(\tau))=0, \quad \forall v, w \in R^{r},
$$

and for all $\tau \in[0, T]$ :

$$
\gamma_{\tau}(v, v)=\hat{\psi}(\tau) \cdot\left[\left[\hat{f}_{\tau}, G v\right], G v\right](\hat{q}(\tau)) \geq 0, \quad \forall v \in R^{r} .
$$

Strong Generalized Legendre Condition (compare with (4.16)) looks like follows: for some $\beta>0$ and for all $\tau \in[0, T]$ :

$$
\gamma_{\tau}(v, v)=\hat{\psi}(\tau) \cdot\left[\left[\hat{f}_{\tau}, G v\right], G v\right](\hat{q}(\tau)) \geq \beta\|v\|^{2}, \quad \forall v \in R^{r} .
$$

Following result is "affine version" of the Proposition 4.3.

Proposition 8.2 (Goh and Legendre Necessary Rigidity Conditions). For an extremal control $\hat{u}(\cdot)$ or the corresponding trajectory $\hat{q}(\cdot)$ of the affine control system (8.1) to be rigid it is necessary, that for some $\hat{\psi}(\cdot)$ the extremal $(\hat{u}(\cdot), \hat{q}(\cdot), \hat{\psi}(\cdot), T)$ meets the Goh condition (8.7) and the Generalized Legendre Condition (8.8).

Let us denote by $\bar{\gamma}_{\tau}$ the nonsingular symmetric $(r \times r)$-matrix, which corresponds to the quadratic form $\gamma_{\tau}(v, v)$ on $R^{r}$. Let $\gamma_{i j}^{-1}(\tau)$ be an $i j$-entry of the matrix $\bar{\gamma}_{\tau}^{-1}$.

Following proposition is "affine version" of the Theorem 4.4.

Proposition 8.3 (Regularity of Extremals for Affine Systems). - If Goh condition (8.7) and Strong Generalized Legendre Condition (8.9) hold along an extremal $(\hat{u}(\cdot), \hat{q}(\cdot), \hat{\psi}(\cdot), T)$ of the control system (8.1), then the extremal control $\hat{u}(\tau)$ is smooth and meets the equality:

$$
\hat{u}_{i}(\tau)=-\sum_{j=1}^{r} \gamma_{i j}^{-1}(\tau) \hat{\psi}(\tau) \cdot\left[f,\left[\hat{f}_{\tau}, g_{j}\right]\right](\hat{q}(\tau)), \quad i=1, \ldots r .
$$


We define correspondingly 1 st and 2 nd variations of the system (8.1) along its extremal $(\hat{u}(\cdot), \hat{q}(\cdot), \hat{\psi}(\cdot), T)$ as the differential and the Hessian of the (time $\times$ input) state mapping $F\left(t, u(\cdot)\right.$ at the point $(T, \hat{u}(\cdot)) \in R \times L_{\infty}^{r}$.

The formulae are: for the first variation

$$
\left.F^{\prime}\right|_{(T, \hat{u}(\cdot))}(\delta \theta, u(\cdot))=\hat{f}_{T}\left(q^{1}\right) \delta \theta+\int_{0}^{T} Y_{\tau}\left(q^{1}\right) u(\tau) d \tau,
$$

and for the second variation

$$
\begin{aligned}
& \left.2 F^{\prime \prime}\right|_{(T, \hat{u}(\cdot))}\left[\hat{\psi}_{T}\right](\delta \theta, u(\cdot)) \\
& \quad=\hat{\psi}_{T} \cdot\left(\int_{0}^{T}\left[-\hat{f}_{T} \delta \theta+\int_{0}^{\tau} Y_{\xi} u(\xi) d \xi, Y_{\tau} u(\tau)\right]\left(q^{1}\right) d \tau\right),
\end{aligned}
$$

with the domain

$$
\left\{(\delta \theta, u(\cdot)) \in\left(R \times L_{\infty}^{r}\right) \mid \hat{f}_{T}\left(q^{1}\right) \delta \theta+\int_{0}^{T} Y_{\tau}\left(q^{1}\right) u(\tau) d \tau=0\right\}
$$

where

$$
Y_{\tau} v=\operatorname{Ad}\left(\overrightarrow{\exp } \int_{T}^{\tau} \hat{f}_{\xi} d \xi\right) G v \quad \forall v \in R^{r}
$$

We define nullity and Morse index of extremal $(\hat{u}(\cdot), \hat{q}(\cdot), \hat{\psi}(\cdot), T)$ as the dimension of the kernel and negative index of the quadratic form (8.11)(8.12). Morse index of extremal path $(\hat{u}(\cdot), \hat{q}(\cdot), T)$ is minimum of the indices of the extremals $(\hat{u}(\cdot), \hat{q}(\cdot), \hat{\psi}(\cdot), T)$, which this path enters.

The following Proposition is direct generalization of the Propositions 4.5 and 4.7.

Proposition 8.4. - If Goh condition (8.7) and Generalized Strong Legendre Condition (8.9) hold along extremal $(\hat{u}(\cdot), \hat{q}(\cdot), \hat{\psi}(\cdot), T)$ of the affine control system (8.1), then:

1) the second variation (8.11)-(8.12) can be extended by continuity onto the space $R \times H_{-1}^{r}[0, T]$ and the extension is weakly positive definite on some subspace of finite codimension in $R \times H_{-1}^{r}[0, T]$, i.e. admits on this subspace a lower estimate:

$$
\left.2 F^{\prime \prime}\right|_{(T, \hat{u}(\cdot))}\left[\hat{\psi}_{T}\right](\delta \theta, u) \geq c\left(\|\left. u\right|_{-1} ^{2}+\delta \theta^{2}\right)
$$

2) the kernel of the second variation is contained in

$$
R \times\left(C^{\infty}\left((0, T), R^{r}\right) \oplus H_{-1}^{r}[0] \oplus H_{-1}^{r}[T]\right) .
$$


The following two theorems are slight generalizations of the obtained in the Section 4 rigidity conditions for vector distributions.

THEOREM 8.5 (Necessary Rigidity Conditions for Affine Systems). - If a corank $k$ extremal path $(\hat{u}(\cdot), \hat{q}(\cdot), T)$ of the system (8.1) is rigid on $[0, T]$, then its Morse index should not exceed $k-1$. In particular for a rigid corank 1 extremal path the index must vanish.

THEOREM 8.6 (Sufficient Rigidity Conditions for Affine Systems). 1) If the second variation along an extremal $(\hat{u}(\cdot), \hat{q}(\cdot), \hat{\psi}(\cdot), T)$ of the affine control system (8.1) is weak positive definite, then the extremal path $(\hat{u}(\cdot), \hat{q}(\cdot), T)$ is rigid. 2) In particular, if Goh condition (8.7) and Strong Generalized Legendre Condition (8.9) hold along the extremal $(\hat{u}(\cdot), \hat{q}(\cdot), \hat{\psi}(\cdot), T)$ and Morse index and nullity of the extremal both vanish, then the extremal path $(\hat{u}(\cdot), \hat{q}(\cdot), T)$ is rigid.

To compute Morse index and nullity which play an important role for the rigidity conditions for extremal paths of affine control system (8.1) we have to repeat almost literally what was done in the Section 5 . We refer to that Section marking only minor differences.

Given an extremal $(\hat{u}(\cdot), \hat{q}(\cdot), \hat{\psi}(\cdot), T)$ of the affine control system (8.1), we define linear space $W$ (compare with (5.1)) as:

$$
W=\operatorname{span}\left\{\left\{\hat{f}_{T}\left(q^{1}\right)\right\} \cup\left\{Y_{\tau}\left(q^{1}\right) v \mid \tau \in[0, T], v \in R^{r}\right\}\right\},
$$

where $\hat{f}_{T}$ and $Y_{\tau}$ are defined by (8.13). Evidently $W=\left.\operatorname{Im} F^{\prime}\right|_{(T, \hat{u}(\cdot))}$ and $\operatorname{codim} W=k$ is corank of the extremal path $(\hat{u}(\cdot), \hat{q}(\cdot), T)$.

Introducing like in the Section 5 the symplectic space $\Sigma(\operatorname{dim} \Sigma=$ $2(n-k))$, Lagrangian plane $\Pi$ and denoting by $\underline{f}$ the canonical projection of the vector field $\hat{f}_{T}$ we see, that (5.4)-(5.5) is as well symplectic representation for the second variation (8.11)-(8.12) of the affine control system (8.1).

Therefore the Jacobi equation for the extremal $(\hat{u}(\cdot), \hat{q}(\cdot), \hat{\psi}(\cdot), T)$ of the affine control system (8.1) has the same form (5.10) or (5.11). Introducing isotropic subspace:

$$
\Gamma_{f}=\operatorname{span}\left\{\underline{f} \cup \underline{G v} \mid v \in R^{r}\right\} \subset \Sigma,
$$

we define Jacobi curve in Lagrangian Grassmanian for the extremal $(\hat{u}(\cdot), \hat{q}(\cdot), \hat{\psi}(\cdot), T)$ (compare with the Definition 5.1).

Definition 8.3 (Jacobi Curve for Extremal of Affine System). - Jacobi curve corresponding to an extremal of the affine control system (8.1) 
is a curve $\tau \mapsto \Lambda_{\tau}(\tau \in[0, T])$ in Lagrangian Grassmanian $\mathcal{L}(\Sigma)$, which coincides for $\tau \in[0, T)$ with the starting at $\Pi$ trajectory of the Jacobi equation (5.11) in $\mathcal{L}(\Sigma)$ and jumps at $T-0$ to $\Lambda_{T}=\Lambda_{T-0}^{\Gamma_{f}}=$ $\Lambda_{T-0} \cap \Gamma_{f}^{b}+\Gamma_{f}$.

As for abnormal sub-Riemannian geodesics Morse index and nullity of the extremal of affine system can be computed via symplectic invariants of the Jacobi curve.

Theorem 8.7 (Index Theorem for Extremals of Affine System). - Let $\tau \mapsto \Lambda_{\tau}(\tau \in[0, T])$, be the Jacobi curve, which corresponds to an extremal $(\hat{u}(\cdot), \hat{q}(\cdot), \hat{\psi}(\cdot), T)$ of the affine control system (8.1). Then for any subdivision $\eta_{s+1}=0=\eta_{0}<\eta_{1}<\cdots<\eta_{s}=T$ of $\tau \mapsto \Lambda_{\tau}$ into simple subarcs $\left.\Lambda\right|_{\left[\eta_{i}, \eta_{i+1}\right]},(i=0, \ldots s-1)$ Morse index of the extremal equals to

$$
\sum_{i=0}^{s} i n d_{\Pi}\left(\Lambda_{\eta_{i}}, \Lambda_{\eta_{i+1}}\right)-(n-k),
$$

where $k$ is the corank of the extremal path $(\hat{u}(\cdot), \hat{q}(\cdot), T)$.

THEOREM 8.8 (Nullity Theorem for Extremals of Affine System). Let the Jacobi curve $\tau \mapsto \Lambda_{\tau}(\tau \in[0, T])$, correspond to an extremal $(\hat{u}(\cdot), \hat{q}(\cdot), \hat{\psi}(\cdot), T)$ of the affine control system (8.1). Then nullity of the extremal, i.e. the dimension of the kernel of the second variation (8.11)(8.12), equals to $\operatorname{dim}\left(\Lambda_{T} \cap \Pi\right)$.

THEOREM 8.9 (Local Rigidity for Extremals of Affine System). - Let an extremal $(\hat{u}(\cdot), \hat{q}(\cdot), \hat{\psi}(\cdot), T)$ of the affine control system (8.1) meet Goh condition (8.7) and Strong Generalized Legendre Condition (8.9). Then for any small enough $\bar{t}>0$ the restrictions $\left(\left.\hat{u}(\cdot)\right|_{[0, \bar{t}]},\left.\hat{q}(\cdot)\right|_{[0, \bar{t}]}, \bar{t}\right)$ of the extremal path $(\hat{u}(\cdot), \hat{q}(\cdot), T)$ on $[0, \bar{t}]$ are rigid.

\section{APPENDIX 1}

\section{ISOLATED POINTS ON CRITICAL LEVELS OF SMOOTH MAPPINGS AND RIGIDITY OF ABNORMAL GEODESICS}

In the Section 3 we have represented the problem of finding minimal geodesics as Lagrange Problem of Calculus of Variations, which is in turn particular case of problem of relative extremum:

$$
\mathcal{J}(x) \longrightarrow \min , F(x)=0,
$$


where $\mathcal{J}$ is a scalar function(al) on a Banach space $X$ and $F$ maps $X$ into $R^{k}$.

Necessary 1st-order condition for local minimality of point $\hat{x} \in X$ for this problem is extremality of $\hat{x}$. Namely, if $\hat{x}$ is point of minimum and $\mathcal{J}, F$ are Frechet differentiable at $\hat{x}$, then there exists a nonzero pair of Lagrange multipliers $\left(\lambda_{0}, \lambda\right) \in R_{+} \times R^{k^{*}}$, such that $\hat{x}$ is critical point for Lagrangian $\mathcal{L}=\lambda_{0} \mathcal{J}(x)+\lambda F(x)$ (Lagrange multipliers rule). We call such $\hat{x}$ extremal point and $\left(\hat{x}, \lambda_{0}, \lambda\right)$ extremal for the problem (9.1).

Evidently an extremal point $\hat{x}$ may enter different extremals with different Lagrange multipliers; corank of an extremal point is the dimension of the space of Lagrange multipliers, which correspond to it.

An extremal $\left(\hat{x}, \lambda_{0}, \lambda\right)$ is normal, whenever $\lambda_{0} \neq 0$, and abnormal otherwise. We use the notation $(\hat{x}, \lambda)$ for abnormal extremals. If $\lambda_{0}=0$, then the functional $\mathcal{J}$ does not at all enter the 1 st-order condition. Since we suppose to deal only with abnormal extremals, then we may at all forget about the functional $\mathcal{J}$ and at once about the words "abnormal" and "extremal". A corank $k$ abnormal extremal point is in fact a corank $k$ critical point of the mapping $F$. We avoid introducing an extra word "rigidity" for phenomenon of isolatedness of point $\hat{x}$ on the level $F^{-1}(0)$ of the mapping $F$, the phenomenon, we suppose to deal with in this Section.

Certainly for $\hat{x}$ to be isolated it is necessary to be critical, since otherwise locally in a small neighborhood of $\hat{x}$ the level $F^{-1}(0)$ is Banach manifold (without isolated points). We are going to set necessary/sufficient conditions for isolatedness of critical point $\hat{x}$ on the critical level $F^{-1}(0)$ of the mapping $F$.

Assuming that $F$ is twice Frechet differentiable at the point $\hat{x}$ we involve into consideration the Hessian of $F$ at the point $\hat{x}$ (see [10]). It is quadratic mapping $F^{\prime \prime}(\hat{x}):\left.\left.\operatorname{ker} F^{\prime}\right|_{\hat{x}} \rightarrow \operatorname{coker} F^{\prime}\right|_{\hat{x}}$. One can represent it as a bundle of quadratic forms

$$
\lambda \mapsto \lambda F^{\prime \prime}(\hat{x})(\xi, \xi),\left.\xi \in \operatorname{ker} F^{\prime}\right|_{\hat{x}},\left.\lambda \perp \operatorname{Im} F^{\prime}\right|_{\hat{x}}
$$

with the domain $\left.\operatorname{ker} F^{\prime}\right|_{\hat{x}}$.

We define index and nullity of $(\hat{x}, \lambda)$ as negative index and dimension of the kernel of the quadratic form $\lambda F^{\prime \prime}(\hat{x})(\xi, \xi)$ on ker $\left.F^{\prime}\right|_{\hat{x}}$. Index of critical point $\hat{x}$ is $\min \left\{\right.$ ind $\left.\lambda F^{\prime \prime}\left|\lambda \perp \operatorname{Im} F^{\prime}\right|_{\hat{x}}\right\}$.

We will show now, that index and nullity provide essential information about local structure of the critical level $F^{-1}(0)$.

Theorem 9.1 (Isolated Points on Critical Levels: Necessary Condition). - Let $X$ be a Banach space and $\hat{x} \in X$ be a corank $m$ critical point for the 
mapping $F: X \rightarrow R^{k}$, which is twice Frechet differentiable at $\hat{x}$. Then for $\hat{x}$ to be an isolated point of the set $F^{-1}(0)$, its index must be less than $m$.

Proof of Theorem 9.1. - We assume without loss of generality, that $\hat{x}$ is the origin of $X$. We denote by $D$ and $h$ correspondingly the differential and the Hessian of the mapping $F$ at the origin. Suppose, that for any $\lambda \in(\operatorname{Im} D)^{\perp}$ index of the quadratic form $\lambda h$ on $\operatorname{ker} D$ is $\geq m$. We are going to prove that then $\hat{x}=0$ is not isolated point of the set $F^{-1}(0)$.

The equation $F(x)=0$ can be represented as a system $f(y, z)=0$, $g(y, z)=0$, where $(y, z)=x$ is such splitting of $x$, that:

(i) $z$ coordinatizes $\operatorname{ker} D, \partial F / \partial z=0$;

(ii) $\operatorname{dim} f=\operatorname{dim} y=\operatorname{rank} D=k-m$,

$$
\operatorname{rank} \partial F /\left.\partial y\right|_{0}=\operatorname{rank} \partial f /\left.\partial y\right|_{0}=\operatorname{rank} D
$$

Then in virtue of Implicit Function Theorem the equation $f(y, z)=0$ can be resolved uniquely w.r.t. $y: y=y(z)$. Substituting $y(z)$ into the equation $g(y, z)=0$ we obtain an equation $\varphi(z)=g(y(z), z)=0$. Obviously $x=0$ is an isolated point of the set $F^{-1}(0)$, if and only if $z=0$ is an isolated point of $\varphi^{-1}(0)$. Let us note, that $\varphi^{\prime}(0)=0$ and hence we may investigate now the mapping $\varphi$, whose differential vanishes at the origin. To avoid additional notation we will assume, instead of it, that $D=\left.F^{\prime}\right|_{0}=0$, and then $h=\left.F^{\prime \prime}\right|_{0}$ is a quadratic mapping of $X$ into $R^{m}$. Again we assume, that for any nonzero $\lambda \in R^{m^{*}}$ index of the quadratic form $\lambda h$ is $\geq m$.

Now we will get rid of infinite-dimensional space $X$.

LEMMA 9.2. - Under the conditions of the Theorem 9.1 there exists a finite-dimensional subspace $W \subset X$, such that for any nonzero $\lambda \in R^{m^{*}}$ index of the quadratic form $\left.\lambda h\right|_{W}$ is $\geq m$.

Proof of Lemma 9.2. - For any unit covector $\bar{\lambda} \in R^{m^{*}}$, there exists a $m$ dimensional subspace $W_{\bar{\lambda}} \subset X$, such that the restriction $\left.\bar{\lambda} h\right|_{W_{\bar{\lambda}}}$ is negative definite. For all $\lambda$ 's from some small neighborhood $\Omega_{\bar{\lambda}}$ of $\bar{\lambda}$ the quadratic forms $\left.\lambda h\right|_{W_{\bar{\lambda}}}$ are also negative definite. Choosing a finite covering of the sphere $\|\lambda\|=1$ by corresponding neighborhoods $\Omega_{\bar{\lambda}^{1}}, \ldots \Omega_{\bar{\lambda}^{-}}$we may take $W=W_{\bar{\lambda}^{1}}+\cdots W_{\bar{\lambda}^{s}}$.

From now on we consider $W$ in place of $X$ or, all the same, assume $\operatorname{dim} X<\infty$.

The following statement enables us to investigate the quadratic mapping $h$ instead of $F$. 
Lemma 9.3. - If the cone $(h)^{-1}(0)$ contains a regular point of the quadratic mapping $h: X \rightarrow R^{m}$, then 0 is not isolated in the set $F^{-1}(0)$.

Proof. - If $y \in h^{-1}(0)$ is a regular point of $h$, then there exists a $m$-dimensional subspace $Z \subset X$, such that $h_{y+Z}:(y+Z) \rightarrow R^{m}$ is local diffeomorphism at $y$. Since $h$ is homogeneous, then the same holds for all points $\eta^{2} y, \eta \neq 0$.

Consider the mapping $\phi_{\epsilon}(z)=h(y+\epsilon z)$, where $z$ belongs to the unit sphere $S^{m-1} \subset Z$. Obviously $h(\eta y+\eta \epsilon z)=\eta^{2} \phi_{\epsilon}(z)$ and, for small enough $\epsilon>0$, the topological degree of the mapping $\phi_{\epsilon} /\left\|\phi_{\epsilon}\right\|: S^{m-1} \rightarrow S^{m-1}$ is +1 or -1 . Since the differentials of $h$ at the points $\eta y$ are nondegenerate, then $\exists a>0$, such that for small enough $\epsilon>0, \forall z \in S^{n-1}$ : $\|h(\eta y+\eta \epsilon z)\| \geq a \eta^{2} \epsilon$.

On the other side

$$
\|F(\eta y+\eta \epsilon z)-h(\eta y+\eta \epsilon z)\|=o\left(\eta^{2}\right)
$$

and therefore for some $\epsilon>0$ and small enough $\eta>0$ topological degree of the mapping

$$
z \longrightarrow F(\eta y+\eta \epsilon z) /\|F(\eta y+\eta \epsilon z)\|
$$

is +1 or -1 . Hence for every small enough $\eta>0$ the equation $F(\eta y+\eta \epsilon z)=0$ has a solution $z_{\eta}$ belonging to the unit ball $B^{n} \subset R^{n}$ and therefore 0 is not isolated point of the set $F^{-1}(0)$.

This Lemma allows us to deal with the quadratic mapping $h$ instead of $F$. The conclusion of the Theorem 4.1 is implied by the following

Proposition 9.4. - Let $P: X \rightarrow R^{m}$ be quadratic mapping $(\operatorname{dim} X<$ $\infty)$, such that ind $\lambda P \geq m, \forall \lambda \in R^{m^{*}} \backslash 0$. Then $P^{-1}(0)$ contains regular point of the mapping $P$.

Proof. - Without loss of generality we may assume, that $\left.P^{\prime}\right|_{x}=0$ if and only if $x=0$. Indeed otherwise the condition $\left.P^{\prime}\right|_{x}=0$ means, that $x$ lies in the intersection of the kernels of the (quadratic forms, which are) components of $P$, and we may take quotient of $X$ w.r.t. to this intersection.

Let us start induction w.r.t. $m$. For $m=1$ the theorem was proved in [7]. Taking $m>1$ we will treat separately two cases.

i) $P^{-1}(0) \neq\{0\}$. Let $P(y)=0$ for some $y \neq 0$. Then $\left.P^{\prime}\right|_{y} \neq 0$ and $\left.\lambda P^{\prime \prime}\right|_{y}$ coincides with the restriction of $\lambda P$ onto $\left.\operatorname{ker} P^{\prime}\right|_{y}\left(\left.\lambda \perp \operatorname{Im} P^{\prime}\right|_{y}\right)$. Conditions of the Proposition imply ind $\left.\lambda P^{\prime \prime}\right|_{y} \geq\left.\operatorname{codim} \operatorname{Im} P^{\prime}\right|_{y}, \forall \lambda \perp$ 
$\left.\operatorname{Im} P^{\prime}\right|_{y}$. Since $\left.\operatorname{dim} \operatorname{coker} P^{\prime}\right|_{y}<m$, then according to the inductive assumption the inverse image $\left(\left.P^{\prime \prime}\right|_{y}\right)^{-1}(0)$ contains regular point and hence $P^{-1}(0)$ contains regular point.

ii) $P^{-1}(0)=\{0\}$. Then $\operatorname{Im} P$ is a closed cone. Applying inductive assumption to the Hessians $\left.P^{\prime \prime}\right|_{y}$ for any $y \neq 0$ we obtain, that $\forall z \in \operatorname{Im} P \backslash 0$ the inverse image $P^{-1}(z)$ contains regular point of $P$ and therefore $\operatorname{Im} P \backslash 0$ is open. This means that $\operatorname{Im} P=R^{m}$.

Let $S$ be unit sphere in $X$. Then $P /\|P\|_{S}: S \rightarrow S^{m-1}$ is a surjective mapping. In virtue of Sard theorem there exists regular value $v$ of this mapping. If $P(x)=a v$ for some $a>0$, then $\operatorname{rank}\left(\left.P\right|_{S}\right)_{x}^{\prime} \geq m-1$. Let $a_{v}=\min \{a>0 \mid a v \in P(S)\} ; a_{v}>0$, since $P^{-1}(0)=0$. Let $x_{v} \in S$ and $P\left(x_{v}\right)=a_{v} v$. The pair $\left(a_{v}, x_{v}\right)$ is point of local minimum and normal extremal point for the following problem of relative extremum:

$$
a \rightarrow \min , P(x)-a v=0 \text {. }
$$

Standard 2nd-order necessary optimality condition for this problem provides existence of $\lambda \in R^{m^{*}} \backslash 0$, such that:

$$
\lambda v \geq 0,\left.\lambda\left(\left.P\right|_{S}\right)^{\prime}\right|_{x_{v}}=0,\left.\quad \lambda\left(\left.P\right|_{S}\right)^{\prime \prime}\right|_{x_{v}} \geq 0 .
$$

Direct computation gives

$$
1 /\left.2 \lambda\left(\left.P\right|_{S}\right)^{\prime \prime}\right|_{x_{v}}(y)=\lambda P(y)-|y|^{2} \lambda P\left(x_{v}\right)
$$

and hence $\lambda P(y) \geq 0$ for all $y \in N=\left\{y\left|y \perp x_{v}, P^{\prime}\right|_{x_{v}} y=0\right\}$. Obviously $N$ is a linear subspace of codimension $m$ in $X$. Since $\lambda P\left(x_{v}\right)=\lambda v \geq 0$ and $x_{v}$ is orthogonal and $P$-orthogonal to $N$, then $\lambda P$ is nonnegative on $N \oplus \operatorname{span}\left\{x_{v}\right\}$ and hence ind $\lambda P \leq m-1$, i.e. we come to a contradiction, which finishes the proofs of the Proposition 9.4 and Theorem 9.1.

Now we are going to derive from the previous theorem the necessary rigidity condition for abnormal geodesics, which was established in the Section 4.

Proof of the Theorem 4.1. - We consider $\{x=(t, u(\cdot)) \in[0, \bar{T}] \times$ $\left.L_{\infty}^{r}[0, T]|| u(t) \mid \equiv 1\right\}, \hat{x}=(T, \hat{u}(\cdot))$, and $F=F(t, u(\cdot))$ be the (time $\times$ input)/state mapping. Since our consideration is local, we may coordinatize small neighborhood of $q^{1}=F\left((T, \hat{u}(\cdot))\right.$ in $M$ by $R^{n}$ and small neighborhood of $(T, \hat{u}(\cdot))$ by $X=[0, \bar{T}] \times L_{\infty}^{r-1}[0, T]$. The (time $\times$ input)/state mapping is not smooth w.r.t. time parameter $t$ but becomes $C^{\ell}$-smooth when restricted onto the space of $C^{\ell}$-smooth controls $u(\cdot)$. Obviously the Hessian of this restriction coincides with the 2 nd 
variation (4.9)-(4.10) and index of the critical point $\hat{x}=(T, \hat{u}(\cdot))$ for this restriction coincides with the one from the Definition 4.4.

Now the Theorem 4.1 follows from the Theorem 9.1.

TheOrem 9.5 (Isolated Points on Critical Levels: Sufficient Condition). - Let Banach $X$ be densely embedded into separable Hilbert space $H: X \hookrightarrow H$. Let a mapping $F: X \rightarrow R^{m}$ be Frechet differentiable at a point $\hat{x} \in X$, which is critical point of $F$, i.e. $\lambda F^{\prime}(\hat{x})=0$ for some nonzero $\lambda \in R^{m^{*}}$. If:

(i) $\left\|F(\hat{x}+x)-F(\hat{x})-F^{\prime}(\hat{x}) x\right\|=o(1)\|x\|_{H}$, as $\|x\|_{X} \rightarrow 0$;

(ii) the function $\lambda F$ admits Taylor expansion at $\hat{x}$ of the form

$$
\lambda F(\hat{x}+x)-\lambda F(\hat{x})=\frac{1}{2} \lambda F^{\prime \prime}(\hat{x})(x, x)+o(1)\|x\|_{H}^{2}, \text { as }\|x\|_{X} \rightarrow 0 .
$$

(iii) the quadratic form $\lambda F^{\prime \prime}(\hat{x})(x, x)$ admits continuous extension from $X$ onto $H$ and is $H$-positive definite on $\operatorname{ker} F^{\prime}(\hat{x})$, i.e. for some $\gamma>0$

$$
\lambda F^{\prime \prime}(\hat{x})(\xi, \xi) \geq 2 \gamma\|\xi\|_{H}^{2}, \quad \forall \xi \in \operatorname{ker} F^{\prime}(\hat{x})
$$

then $\hat{x}$ is an isolated point of the level set $F^{-1}(F(\hat{x}))$.

Proof of the Theorem 9.5. - Without loss of generality we may assume, that $F(\hat{x})=0$ and $\hat{x}$ is the origin of $X$. We are going to establish, that $\|F(x)\| \geq \mu\|x\|_{H}^{2}$ for some $\mu>0$ and all $x$ from some small neighborhood of the origin in $X$.

Let us take any linear complement $Z$ to $\operatorname{ker} F^{\prime}(0) ; F^{\prime}(0)$ maps the finite-dimensional subspace $Z$ onto the image $F^{\prime}(0) X$ isomorphically. Any $x \in X$ can be represented uniquely as a sum $x=x_{1}+x_{0}$ of $x_{1} \in Z, x_{0} \in \operatorname{ker} F^{\prime}(0)$, and for some $c>0$

$$
\left\|F^{\prime}(0) x\right\| \geq c\left\|x_{1}\right\|_{H}, \quad \forall x \in X .
$$

For the Lagrange multiplier $\lambda \in R^{m^{*}}$ let us define $N=\left\{y \in R^{m} \mid \lambda y=\right.$ $0\}$ and choose a vector $\eta \in R^{m}$ such, that $\lambda \eta=1$. Then $R^{m}=R \eta+N$ and $\operatorname{Im} F^{\prime}(0) \subseteq N$.

We may present $F(x)$ as a sum of two addends $(\lambda F(x)) \eta$ and the rest $F_{N}(x)$ with values lying in $N$. The norm $\|F(x)\|$ admits an estimate $\|F(x)\| \geq a\left(|\lambda F(x)|+\left\|F_{N}(x)\right\|\right)$ for some $a>0$. In virtue of (9.2)

$$
F_{N}(x)=F^{\prime}(0) x_{1}+o(1)\left(\left\|x_{0}\right\|_{H}+\left\|x_{1}\right\|_{H}\right), \text { as }\|x\|_{X} \rightarrow 0 .
$$


The condition (9.3) and the continuity of the quadratic form $\lambda F^{\prime \prime}(\hat{x})(x, x)$ in the norm of $H$ imply

$$
\lambda F(x)=\frac{1}{2} \lambda F^{\prime \prime}(\hat{x})\left(x_{0}, x_{0}\right)+O(1)\|x\|_{H}\left\|x_{1}\right\|_{H}+o(1)\|x\|_{H}^{2} .
$$

Since $\|x\|_{H}^{2}=O(1)\left(\|x\|_{H}\left\|x_{1}\right\|_{H}+\left\|x_{0}\right\|_{H}^{2}\right)$, we can transform the last estimate into

$$
\lambda F(x)=\frac{1}{2} \lambda F^{\prime \prime}(\hat{x})\left(x_{0}, x_{0}\right)+O(1)\|x\|_{H}\left\|x_{1}\right\|_{H}+o(1)\left\|x_{0}\right\|_{H}^{2} .
$$

Fixing positive $\epsilon<\min (c, \gamma)$ we may choose in virtue of (9.6)-(9.7) a small neighborhood $V$ in $X$ such that for some positive $k$ and any $x \in V$ :

$$
\left\|F_{N}(x)-F^{\prime}(0) x_{1}\right\| \leq \epsilon\left(\left\|x_{0}\right\|_{H}+\left\|x_{1}\right\|_{H}\right)
$$

and

$$
\left|\lambda F(x)-\frac{1}{2} \lambda F^{\prime \prime}(\hat{x})\left(x_{0}, x_{0}\right)\right| \leq k\|x\|_{H}\left\|x_{1}\right\|_{H}+\epsilon\left\|x_{0}\right\|_{H}^{2} .
$$

Together with (9.4) and (9.5) this implies

$$
\left\|F_{N}(x)\right\| \geq \max \left(0,(c-\epsilon)\left\|x_{1}\right\|_{H}-\epsilon\left\|x_{0}\right\|_{H}\right)
$$

and

$$
|\lambda F(x)| \geq \max \left(0,(\gamma-\epsilon)\left\|x_{0}\right\|_{H}^{2}-k\|x\|_{H}\left\|x_{1}\right\|_{H}\right) .
$$

Denoting $c=c-\epsilon, \gamma=\gamma-\epsilon$ we obtain

$\|F(x)\| \geq a\left(\max \left(0, c\left\|x_{1}\right\|_{H}-\epsilon\left\|x_{0}\right\|_{H}\right)\right)+\max \left(0, \gamma\left\|x_{0}\right\|_{H}^{2}-\left.k\|x\|_{H}\left\|x_{1}\right\|\right|_{H}\right)$.

Without loss of generality we may assume that $4 \epsilon k(1+4 \epsilon / c) \leq \gamma c / 2$.

Now if $c\left\|x_{1}\right\|_{H} \geq 4 \epsilon\left\|x_{0}\right\|_{H}$ then

$$
\|F(x)\| \geq a\left(\frac{c}{2}\left\|x_{1}\right\|_{H}+\epsilon\left\|x_{0}\right\|_{H}\right) \geq \alpha(\epsilon, c)\|x\|_{H}^{2}
$$

with $\alpha(\epsilon, c)>0$.

Otherwise if $c\left\|x_{1}\right\|_{H} \leq 4 \epsilon\left\|x_{0}\right\|_{H}$ then

$$
\begin{aligned}
\|F(x)\| & \geq a\left(\gamma\left\|x_{0}\right\|_{H}^{2}-k\|x\|_{H}\left\|x_{1}\right\|_{H}\right) \\
& \geq a\left(\gamma\left\|x_{0}\right\|_{H}^{2}-\left\|x_{0}\right\|_{H}^{2} k(1+4 \epsilon / c) 4 \epsilon / c\right) \\
& \geq(a \gamma / 2)\left\|x_{0}\right\|_{H}^{2} \geq \frac{a \gamma}{2(1+4 \epsilon / c)^{2}}\|x\|_{H}^{2} .
\end{aligned}
$$

Basing on the Theorem 9.5 we shall prove the Theorem 4.8, which provides sufficient rigidity condition for abnormal geodesics. 
Proof of the Theorem 4.8. - We will verify the assumptions of the Theorem 9.5 for the (timexinput)/state mapping $F$. Since our consideration is local i.e. regards small neighborhoods of $q^{1} \in M$ of $(T, \hat{u}(\cdot)) \in$ $\left\{(t, u(\cdot)) \in[0, \bar{T}] \times L_{\infty}^{r}[0, T]|| u(t) \mid \equiv 1\right\}$ then coordinatizing these neighborhoods by $R^{n}$ and $X=[0, \bar{T}] \times L_{\infty}^{r-1}[0, T]$ correspondingly, taking $H=[0, \bar{T}] \times H_{-1}^{r-1}[0, T], \quad x=(t, u(\cdot)) ; \hat{x}=(T, \hat{u}(\cdot)), \lambda=\hat{\psi}_{T}$ and $F=F(t, u(\cdot))$ being the (time $\times$ input)/state mapping.

If $(T, \hat{u}(\cdot))$ enters an abnormal geodesic $(\hat{u}(\cdot), \hat{q}(\cdot), \hat{\psi}(\cdot), T)$, then $(T, \hat{u}(\cdot))$ is a critical point of $F$ and $\hat{\psi}_{T} \in R^{n^{*}}$ (see above) is an annihilator of $\left.\operatorname{Im} F^{\prime}\right|_{(T, \hat{u}(\cdot))}$.

Let us put

$$
\|(t, u(\cdot))\|_{\infty}=|t|+\|u(\cdot)\|_{L_{\infty}},\|(t, u(\cdot))\|_{-1}=\left(|t|^{2}+\|u(\cdot)\|_{H_{-1}}^{2}\right)^{1 / 2}
$$

and extend $\hat{u}(t)$ smoothly onto $[0, T+1]$. The condition (9.4) is fullfilled by virtue of the positive definiteness of the 2 nd variation.

To verify other conditions it is useful to introduce another representation of $F$. Recall that

$$
\begin{aligned}
F & (T+\theta, \hat{u}(\cdot)+u(\cdot)) \\
& =q^{0} \circ \overrightarrow{\exp } \int_{0}^{T+\theta} G \hat{u}(\tau) d \tau \circ \overrightarrow{\exp } \int_{0}^{T+\theta} Y_{T+\theta, \tau} u(\tau) d \tau \\
& =q^{1} \circ \overrightarrow{\exp } \int_{T}^{T+\theta} G \hat{u}(\tau) d \tau \circ \overrightarrow{\exp } \int_{0}^{T+\theta} Y_{T+\theta, \tau} u(\tau) d \tau,
\end{aligned}
$$

where $Y_{t, \tau} v=\overrightarrow{\exp } \int_{t}^{\tau} \operatorname{ad} G \hat{u}(\xi) d \xi G v$.

Now we shall transform the chronological exponential $\overrightarrow{\exp } \int_{0}^{T+\theta} Y_{T+\theta, \tau} u(\tau) d \tau$ by means of the integration by parts formula for a chronological exponential, established in [21]. We derive:

$$
\begin{gathered}
F(T+\theta, \hat{u}(\cdot)+u(\cdot))=q^{1} \circ \overrightarrow{\exp } \int_{T}^{T+\theta} G \hat{u}(\tau) d \tau \circ \\
\overrightarrow{\exp } \int_{0}^{T+\theta}\left(\left(I-\int_{0}^{1} e^{(1-\eta) \operatorname{ad} Y_{T+\theta, \tau} v(\tau)} d \eta\right) Y_{T+\theta, \tau} u(\tau)\right. \\
\left.-\int_{0}^{1} e^{(1-\eta) \operatorname{ad} Y_{T+\theta, \tau} v(\tau)} d \eta \dot{Y}_{T+\theta, \tau} v(\tau)\right) d \tau \circ e^{G v(T+\theta)}
\end{gathered}
$$

where $v(\cdot)=\int_{0}^{\cdot} u(\xi) d \xi$ and $\dot{Y}$ denotes the partial derivative $\partial Y_{t, \tau} / \partial \tau$. 
Taking Volterra expansions for $\overrightarrow{\exp } \int_{T}^{T+\theta} G \hat{u}(\tau) d \tau$ and for the ordinary and chronological exponentials in the last formula we derive

$$
\begin{aligned}
& F(T+\theta, \hat{u}(\cdot)+u(\cdot))=q^{1}+q^{1} \circ\left(\hat{f}_{T} \theta-\int_{0}^{T} \dot{Y}_{T+\theta, \tau} v(\tau) d \tau+G v(T)\right) \\
& \quad+o(\mathbf{1})\left(|\theta|+|v(T)|+\|v(\cdot)\|_{L_{2}}\right), \text { as }\|(\theta, u(\cdot))\|_{\infty} \rightarrow 0,
\end{aligned}
$$

from where the estimate (9.2) follows directly.

To verify the estimate (9.3) we will multiply (9.8) by $\lambda=\hat{\psi}_{T}$ and simplify it getting rid of the terms which are more than quadratic in $v(\cdot)$ and therefore admit an estimate $o(1)\|u(\cdot)\|_{-1}^{2}$. We obtain

$$
\begin{aligned}
& \hat{\psi}_{T}\left(F(T+\theta, \hat{u}(\cdot)+u(\cdot))-q^{1}\right)=\hat{\psi}_{T} q^{1} \circ \overrightarrow{\exp } \int_{T}^{T+\theta} G \hat{u}(\tau) d \tau \circ \\
& \quad \circ\left(-I+\overrightarrow{\exp } \int_{0}^{T+\theta}-\left[Y_{T+\theta, \tau} v(\tau), Y_{T+\theta, \tau} u(\tau)\right]\right. \\
& \left.\quad-\dot{Y}_{T+\theta, \tau} v(\tau)+\left[\dot{Y}_{T+\theta, \tau} v(\tau), Y_{T+\theta, \tau} v(\tau)\right] d \tau \circ e^{G v(T+\theta)}\right) \\
& \quad+o(\mathbf{1}) \|\left(\theta,\left.u(\cdot)\right|_{-1} ^{2} .\right.
\end{aligned}
$$

Recall that Goh condition $\forall u, v \in R^{r} \hat{\psi}_{T}\left[Y_{T, \tau} v, Y_{T, \tau} u\right]\left(q^{1}\right)=0$ is necessary for weak positive definiteness of the second variation. Taking it into account together with the equalities

$$
\hat{\psi}_{T} G\left(q^{1}\right) u=0 ; \hat{\psi}_{T} Y_{T, \tau}\left(q^{1}\right) u=\hat{\psi}_{T} \dot{Y}_{T, \tau}\left(q^{1}\right) v=0
$$

we consider the Volterra expansions of the chronological exponentials in the last formula deriving

$$
\begin{aligned}
& \hat{\psi}_{T}\left(F(T+\theta, \hat{u}(\cdot)+u(\cdot))-q^{1}\right)=\hat{\psi}_{T}\left(\hat{f}_{T} \circ \hat{f}_{T} \theta^{2} / 2\right. \\
& \quad-\left.\theta \int_{0}^{T} \frac{\partial}{\partial \theta}\right|_{\theta=0} \dot{Y}_{T+\theta, \tau} v(\tau) d \tau \\
& \quad+\int_{0}^{T}\left[\dot{Y}_{T, \tau} v(\tau), Y_{T, \tau} v(\tau)\right] d \tau+\theta \hat{f}_{T} \circ \int_{0}^{T} \dot{Y}_{T, \tau} v(\tau) d \tau \\
& +\int_{0}^{T} \int_{0}^{t} \dot{Y}_{T, \tau} v(\tau) d \tau \circ \dot{Y}_{T, t} v(t) d t \\
& \left.+\theta \hat{f}_{T} \circ G v(T)+\frac{1}{2} G v(T) \circ G v(T)\right)\left(q^{1}\right) \\
& +O\left(\|(\theta, u(\cdot))\|_{\infty}\|(\theta, u(\cdot))\|_{-1}^{2}\right) .
\end{aligned}
$$


The rest term contains terms of order $>2$ in $(\theta, u(\cdot), v(\cdot))$ of the Volterra expansions, while the specified terms form $\left.\frac{1}{2} \hat{\psi}_{T} F\right|_{(T, \hat{u})} ^{\prime \prime}(\theta, u(\cdot))$.

The last formula provides the estimate (9.3) for the (time $\times$ input)/state mapping $F$ and implies the continuity of the quadratic form $\lambda F^{\prime \prime}(\hat{x})(x, x)$ in $H$. The statement of the theorem follows now from the Theorem 9.5 .

\section{APPENDIX 2}

\section{GOH CONDITION AND (FIRST) GENERALIZED LEGENDRE CONDITION}

In this Section we provide a proof of the announced in the Section 4 necessity of the Goh condition (4.11) and the Generalized Legendre Condition (4.15) for the finiteness of index of the 2 nd variation (4.9)(4.10), or, all the same, of its symplectic representation (5.4)-(5.5). Since we consider bounded measurable controls unlike piecewise-smooth controls treated in the earlier papers, then we find it worth giving here a self-contained proof.

We shall work with the symplectic representation (5.4)-(5.5) of the 2 nd variation

$$
\left.2 F^{\prime \prime}\right|_{\left[T, \hat{u}(\cdot), \psi_{T}\right]}(\delta \theta, u(\cdot))=\int_{0}^{T} \sigma\left(-\hat{g} \delta \theta+\int_{0}^{\tau} \Upsilon_{\xi} u(\xi) d \xi, \Upsilon_{\tau} u(\tau)\right) d \tau
$$

whose domain is:

$$
\left\{(\delta \theta, u(\cdot)) \in R \times L_{\infty}^{r} \mid \hat{g} \delta \theta+\int_{0}^{T} \Upsilon_{\tau} u(\tau) d \tau \in \Pi\right\}
$$

Recall that the vector functions $\tau \mapsto \Upsilon_{\tau}$ are Lipschitzian and hence differentiable for almost all $\tau \in[0, T]$, in fact, for all $\tau$, which are the Lebesgue points of $\tau \mapsto \hat{u}(\tau)$.

Let us consider the depending on $\tau \in[0, T]$ bilinear forms

$$
\sigma\left(\Upsilon_{\tau} u, \Upsilon_{\tau} v\right), \quad u, v \in R^{r}, \quad \tau \in[0, T]
$$

and the quadratic forms

$$
\sigma\left(\dot{\Upsilon}_{\tau} v, \Upsilon_{\tau} v\right), \quad v \in R^{r}, \quad \tau \in[0, T]
$$

defined for almost all $\tau \in[0, T]$.

Vol. 13, $\mathrm{n}^{\circ}$ 6-1996. 
PROPOSITION 10.1. - For the quadratic form (10.1)-(10.2) to have finite negative index the bilinear forms (10.3) must vanish for all $\tau \in[0, T]$ and the quadratic forms (10.4) must be nonnegative for almost all $\tau \in[0, T]$.

Proof. - First we shall restrict the 2 nd variation (10.1)-(10.2) onto the subspace of codimension 1 in (10.2), defined by the equality $\delta \theta=0$. Evidently the index of the new form may differ at most by 1 from the index of the original form. Therefore from now we work with the reduced quadratic form

$$
u(\cdot) \mapsto \int_{0}^{T} \sigma\left(\int_{0}^{\tau} \Upsilon_{\xi} u(\xi) d \xi, \Upsilon_{\tau} u(\tau)\right) d \tau
$$

with the domain:

$$
\left.\left\{u(\cdot) \in L_{\infty}^{r}[0, T]\right] \int_{0}^{T} \Upsilon_{\tau} u(\tau) d \tau \in \Pi\right\},
$$

which coincides with the 2nd variation treated in [1].

Then we shall consider the quadratic form (10.5)-(10.6) for those variations $u(\cdot)$, whose supports are located on a (small) subinterval $[t, t+s] \subset[0, T]$, where $s>0$ and $t$ is a Lebesgue point of $\tau \mapsto \Upsilon_{\tau}$. Integrating (10.5)-(10.6) by parts we obtain

$$
\begin{aligned}
\int_{t}^{t+s} & \sigma(\int_{t}^{\tau} \Upsilon_{\xi} \underbrace{u(\xi) d \xi}_{d v}, \Upsilon_{\tau} u(\tau)) d \tau \\
= & \int_{t}^{t+s} \sigma\left(\Upsilon_{\tau} v(\tau), \Upsilon_{\tau} u(\tau)\right) d \tau-\int_{t}^{t+s} \sigma(\int_{t}^{\tau} \dot{\Upsilon}_{\xi} v(\xi) d \xi, \Upsilon_{\tau} \underbrace{u(\tau)) d \tau}_{d v} \\
= & \int_{t}^{t+s} \sigma\left(\Upsilon_{\tau} v(\tau), \Upsilon_{\tau} u(\tau)\right) d \tau \\
& +\int_{t}^{t+s} \sigma\left(\dot{\Upsilon}_{\tau} v(\tau), \Upsilon_{\tau} v(\tau)\right) d \tau+\sigma\left(\Upsilon_{t+s} v(t+s), \int_{t}^{t+s} \dot{\Upsilon}_{\xi} v(\xi) d \xi\right) \\
& +\int_{t}^{t+s} \sigma\left(\int_{t}^{\tau} \dot{\Upsilon}_{\xi} v(\xi) d \xi, \dot{\Upsilon}_{\tau} v(\tau)\right) d \tau,
\end{aligned}
$$

where $v(\tau)=\int_{t}^{\tau} u(\xi) d \xi$ and

$$
\Upsilon_{t+s} v(t+s)-\int_{t}^{t+s} \dot{\Upsilon}_{\tau} v(\tau) d \tau \in \Pi
$$


Let us restrict once more the 2nd variation on the set of $u(\cdot)$, which satisfy the condition $\int_{t}^{t+s} u(\xi) d \xi=0$. This subspace has codimension $r$ in the space of all $u(\cdot)$ 's with supports in $[t, t+s]$. The 2 nd variation takes on this subspace the form

$$
\begin{aligned}
& \int_{t}^{t+s} \sigma\left(\Upsilon_{\tau} v(\tau), \Upsilon_{\tau} u(\tau)\right) d \tau+\int_{t}^{t+s} \sigma\left(\dot{\Upsilon}_{\tau} v(\tau), \Upsilon_{\tau} v(\tau)\right) d \tau \\
& \quad+\int_{t}^{t+s} \sigma\left(\int_{t}^{\tau} \dot{\Upsilon}_{\xi} v(\xi) d \xi, \dot{\Upsilon}_{\tau} v(\tau)\right) d \tau
\end{aligned}
$$

The subspace

$$
E_{s}=\left\{u(\cdot) \in L_{\infty}^{r}[t, t+s]: \int_{t}^{t+s} \dot{\Upsilon}_{\tau} v(\tau) d \tau \in \Pi, v(t+s)=0\right\}
$$

is the domain of this form, which is a subspace of codimension no more than $m=n-k+r$ in $L_{\infty}^{r}[0, T]$, with $k$ being the corank of our abnormal geodesic.

Introducing the time stretching $(\tau-t) / s=\theta$, we transform the integral quadratic form (10.7)-(10.8) into

$$
\begin{aligned}
& \int_{0}^{1} \sigma\left(\Upsilon_{t+s \theta} v(t+s \theta), \Upsilon_{t+s \theta} u(t+s \theta)\right) s d \theta \\
& \quad+\int_{0}^{1} \sigma\left(\dot{\Upsilon}_{t+s \theta} v(t+s \theta), \Upsilon_{t+s \theta} v(t+s \theta)\right) s d \theta \\
& \quad+\int_{0}^{1} \sigma\left(\int_{0}^{\theta} \dot{\Upsilon}_{t+s \xi} v(t+s \xi) s d \xi, \dot{\Upsilon}_{t+s \theta} v(t+s \theta)\right) s d \tau .
\end{aligned}
$$

Let us introduce the notation $u_{s}(\theta)=u(t+s \theta)$ and $v_{s}(\theta)=$ $\int_{0}^{\theta} u_{s}(\xi) d \xi, \theta \in[0,1]$; then $v(t+s \theta)=s v_{s}(\theta)$ and the quadratic form can be represented as

$$
\begin{aligned}
& s^{2} \int_{0}^{1} \sigma\left(\Upsilon_{t+s \theta} v_{s}(\theta), \Upsilon_{t+s \theta} u_{s}(\theta)\right) d \theta \\
& \quad+s^{3} \int_{0}^{1} \sigma\left(\dot{\Upsilon}_{t+s \theta} v_{s}(\theta), \Upsilon_{t+s \theta} v_{s}(\theta)\right) d \theta \\
& \quad+s^{4} \int_{0}^{1} \sigma\left(\int_{0}^{\theta} \dot{\Upsilon}_{t+s \xi} v_{s}(\xi) d \xi, \dot{\Upsilon}_{t+s \theta} v_{s}(\theta)\right) d \tau \\
& \quad=s^{2} \int_{0}^{1} \sigma\left(\Upsilon_{t} v_{s}(\theta), \Upsilon_{t} u_{s}(\theta)\right) d \theta+o\left(s^{2}\right), \text { as } s \rightarrow 0 .
\end{aligned}
$$


Consider the principal term

$$
G_{t}\left(u_{s}(\cdot)\right)=\int_{0}^{1} \sigma\left(\Upsilon_{t} v_{s}(\theta), \Upsilon_{t} u_{s}(\theta)\right) d \theta
$$

The involution $J: u_{s}(\theta) \mapsto u_{s}(1-\theta)$ changes the sign of $G_{t}$ : $G_{t}\left(J u_{s}(\cdot)\right)=-G_{t}\left(u_{s}(\cdot)\right)$.

The existence of such involution implies, that for any subspace in $L_{\infty}^{r}[0,1]$, on which the quadratic form $(10.10)$ is positive definite, there exists a subspace of the same dimension on which it is negative definite. Were the negative index of the form finite, we would obtain contradiction with the evident fact, that the kernel of the form (10.10) has infinite codimension in $L_{\infty}^{r}[0, T]$.

Therefore, if the Goh condition is violated at the point $t$, then the quadratic form $G_{t}$ (which does not depend on $s$ ) has infinite negative index. Take arbitrary integer $N$ and an $m+N$-dimensional subspace $A_{N}$ of $L_{\infty}^{r}[0,1]$ such that $\left.G_{t}\right|_{A_{N}}<0$. This form is even negative definite, since $A_{N}$ is finitedimensional. Then by virtue of the representation (10.9) for the restriction of the 2nd variation on $E_{s}$, we derive that for small enough $s>0$ the 2nd variation is negative on the subspace $A_{N} \cap E_{s}$ with the dimension $\geq N$.

Returning to the expression (10.9) and assuming the Goh condition to hold on $[t, t+s]$ we may represent the 2 nd variation as

$$
\begin{aligned}
& s^{3} \int_{0}^{1} \sigma\left(\dot{\Upsilon}_{t+s \theta} v_{s}(\theta), \Upsilon_{t+s \theta} v_{s}(\theta)\right) d \theta \\
& \quad+s^{4} \int_{0}^{1} \sigma\left(\int_{0}^{\theta} \dot{\Upsilon}_{t+s \xi} v_{s}(\xi) d \xi, \dot{\Upsilon}_{t+s \theta} v_{s}(\theta)\right) d \tau
\end{aligned}
$$

If $t$ is a Lebesgue point of $t \mapsto \hat{u}(t)$ and therefore also of $t \mapsto \dot{\Upsilon}_{t}$, then (10.11) can be represented as

$$
s^{3} \int_{0}^{1} \sigma\left(\dot{\Upsilon}_{t} v_{s}(\theta), \Upsilon_{t} v_{s}(\theta)\right) d \theta+o\left(s^{3}\right)\left\|v_{s}(\cdot)\right\|_{L_{\infty}}^{2}, \text { as } s \rightarrow 0
$$

Evidently if the Generalized Legendre Condition fails, i.e. $\sigma\left(\dot{\Upsilon}_{t} v_{0}, \Upsilon_{t} v_{0}\right)<0$ for some $v_{0} \in R^{r}$, then for arbitrarily large $N$ there exists a $m+N$-dimensional subspace $A_{N}^{\prime}$ consisting of the functions of the form $\alpha(\cdot) v_{0}$ with $\alpha(\cdot) \in L_{\infty}[0, T]$, on which the quadratic form $\int_{0}^{1} \sigma\left(\dot{\Upsilon}_{t} v_{s}(\theta), \Upsilon_{t} v_{s}(\theta)\right) d \theta$ is negative. Then for small enough $s>0$ the 2nd variation, admitting the representation (10.12), is negative on the subspace $A_{N}^{\prime} \cap E_{s}$ with the dimension $\geq N$. 


\section{REFERENCES}

[1] A. A. Agrachev, Quadratic Mappings in Geometric Control Theory, in: Itogi Nauki $i$ Tekhniki; Problemy Geometrii, VINITI, Acad. Nauk SSSR, Moscow, Vol. 20, pp. 11-205, 1988. English transl. in J. Soviet Math., Vol. 51, 1990, pp. 2667-2734.

[2] A. A. Agrachev, Topology of Quadratic Mappings and Hessians of Smooth Mappings, in: Itogi Nauki i Tekhniki; Algebra, Topologia, Geometria; VINITI, Acad. Nauk SSSR, Vol. 26, 1988, pp. 85-124.

[3] A. A. Agrachev, The Second-Order Optimality Condition in the General Nonlinear Case, Matem Sbornik, Vol. 102, 1977, pp. 551-568. English transl. in: Math. USSR Sbornik, Vol. 31, 1977.

[4] A. A. Agrachev and R. V. Gamkrelidze, Second-Order Optimality Condition for the Time-Optimal Problem, Matem. Sbornik, Vol. 100, 1976, pp. 610-643. English transl. in: Math. USSR Sbornik, Vol. 29, 1976, pp. 547-576.

[5] A. A. Agrachev and R. V. Gamkrelidze, Exponential Representation of Flows and Chronological Calculus, Matem. Sbornik, Vol. 107, 1978, pp. 467-532. English transl. in: Math. USSR Sbornik, Vol. 35, 1979, pp. 727-785.

[6] A. A. Agrachev, R. V. Gamkrelidze and A. V. Sarychev, Local Invariants of Smooth Control Systems, Acta Applicandae Mathematicae, Vol. 14, 1989, pp. 191-237.

[7] A. A. Agrachev and A. V. SARYCHEV, On Abnormal Extremals for Lagrange Variational Problems, to appear in J. Mathematical Systems, Estimation and Control.

[8] A. A. Agrachev and A. V. SARYChev, On Abnormal Extremals for Lagrange Variational Problems (summary), J. Mathematical Systems, Estimation and Control, Vol. 5, 1995, pp. 127-130.

[9] V. I. ARnol'D, Mathematical Methods of Classical Mechanics, Springer-Verlag, New York-Berlin, 1978.

[10] V. I. Arnol'd, A. N. Varchenko and S. M. GuSein-Zade, Singularities of Differentiable Mappings, Vol. 1, Birkhäuser, Boston, 1985.

[11] B. BONNARD and I. KUPKA, Théorie de singularités de l'application entree/sortie et optimalité des trajectoires singulières dans le problème du temps minimal, Preprint, 1990.

[12] V. GershKovich, Engel Structures on Four Dimensional Manifolds, University of Melbourne, Department of Mathematics, Preprint Series No. 10, 1992.

[13] B. S. GoH, Necessary Conditions for Singular Extremals Involving Multiple Control Variables, SIAM J. Control, Vol. 4, 1966, pp. 716-731.

[14] V. Guillemin and S. STernberg, Geometric Asymptotics, Amer. Math. Soc., Providence, Rhode Island, 1977.

[15] G. W. Haynes and H. Hermes, Nonlinear Controllability via Lie Theory, SIAM J. on Control, Vol. 8, 1970 , pp. 450-460.

[16] H. J. Kelley, R. Kopp and H. G. Moyer, Singular Extremals, in: G. Leitman Ed., Topics in Optimization, Academic Press, New York, N. Y., 1967, pp. 63-101.

[17] A. J. KRENER, The High-Order Maximum Principle and its Applications to Singular Extremals, SIAM J. on Control and Optimiz., Vol. 15, 1977, pp. 256-293.

[18] G. LION and M. VERGne, The Weyl Representation, Maslov Index and Theta-series, Birkhäuser, Boston, 1980.

[19] R. Montgomery, Geodesics, Which Do Not Satisfy Geodesic Equations, Preprint, 1991.

[20] M. Morse, The Calculus of variations in the large, Amer. Math. Soc., New York, NY, 1934.

[21] A. V. SARYCHEV, Integral representation for the trajectories of control system with generalized right-hand side (in Russian), Differentsialnye Uravnenia, Vol. 24, 1988, pp. 1551-1564. English transl.: Differential Equations, Vol. 24, 1988, pp. 1021-1031.

[22] A. V. SARYCHEV, The index of the second variation of the control system, Matem. Sbornik, Vol. 113, 1980, pp. 464-486. English transl. in: Math. USSR Sbornik, Vol. 41, 1982, pp. 383-401.

[23] H. J. SussmanN, A Cornucopia of Abnormal Sub Riemannian Minimizers. Part I: the Four-Dimensional Case, IMA Preprint Series \#1073,1992.

Vol. $13, \mathrm{n}^{\circ}$ 6-1996. 
[24] H. J. Sussmann and W. Liu, Shortest Paths for Sub-Riemannian Metrics on Rank-2 Distributions, Preprint, 1993.

[25] L. C. Young, Lectures on the Calculus of Variations and Optimal Control Theory, Chelsea, New York, 1980.

[26] A. A. Agrachev and A. V. Sarychev, Strong Minimality of Abnormal Geodesics for 2-Distributions, J. of Dynamical and Control Systems, Vol. 1, 1995, pp. 139-176.

(Manuscript received December 15, 1993;

revised February 14, 1995.) 